$$
\begin{aligned}
& \text { CADRE } \\
& \text { RÉGLEMENTAIRE }
\end{aligned}
$$

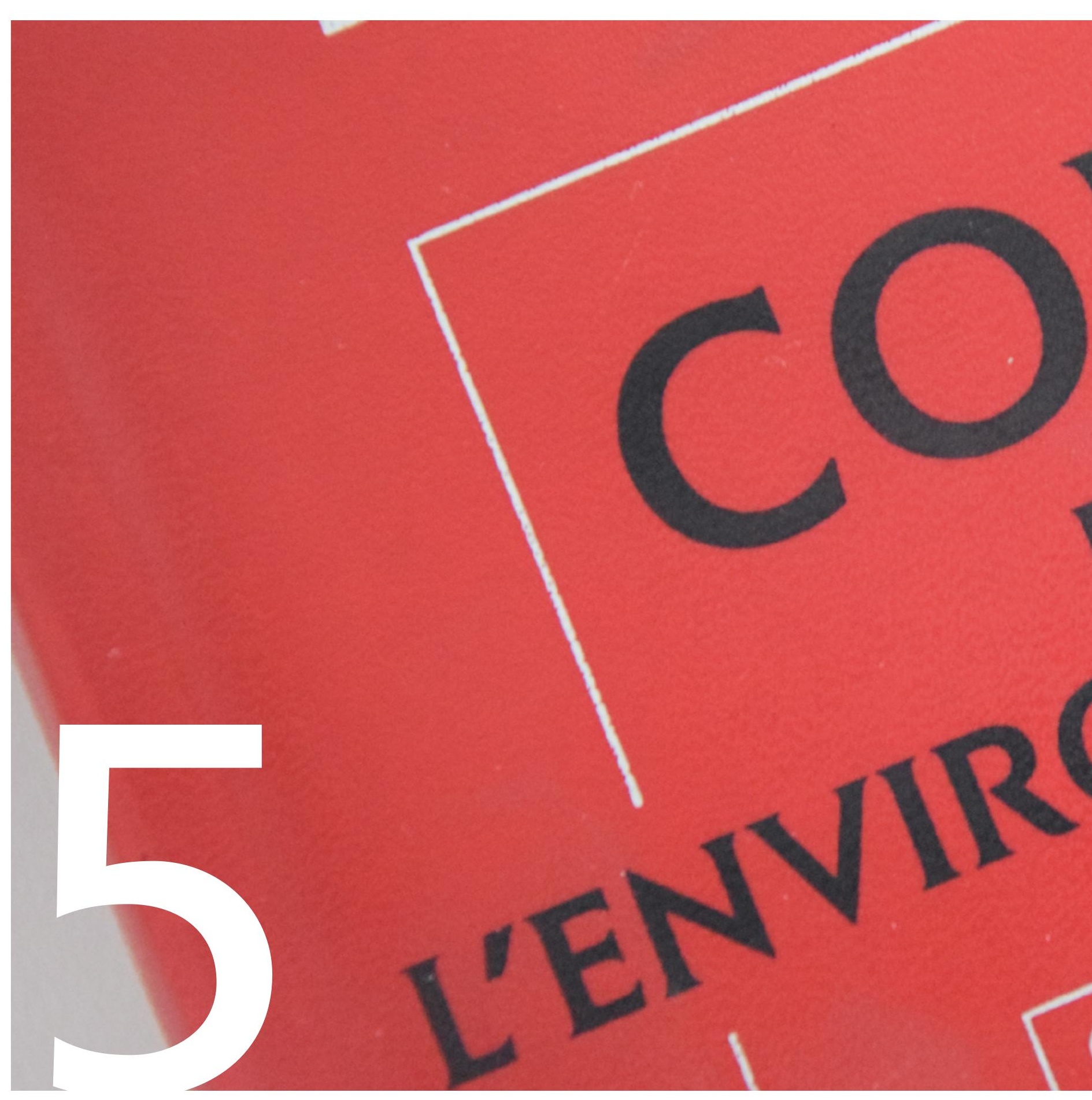




\section{CADRE GÉNÉRAL}

2. DROIT INTERNATIONAL DE L'ENVIRONNEMENT

2.1 Organismes internationaux et organisations non gouvernementales concernés

2.2 Principaux textes internationaux ratifiés par la France

- Convention de Barcelone sur la protection du littoral méditerranéen, 1976

- Protocole de Montréal relatif à des substances qui appauvrissent la couche d'ozone, 1987

- Convention OSPAR sur la protection du milieu marin de l'Atlantique Nord-Est, 1992

- Protocole de Kyoto sur la réduction des gaz à effet de serre, 1997

- Convention de Berne pour la protection du Rhin, 1999

- Accord international de Gand sur la Meuse, 2002

\section{RÉGLEMENTATION EUROPÉENNE}

3.1 Textes relatifs à la protection de la santé publique

- Le Traité EURATOM, 1957

- Directive 96/26 Euratom sur les normes de base en radioprotection abrogée par la directive 2013/59/Euratom

- Directive 98/83/CE sur la qualité des eaux destinées à la consommation humaine

- Directive 2006/7/CE sur la qualité des eaux de baignade

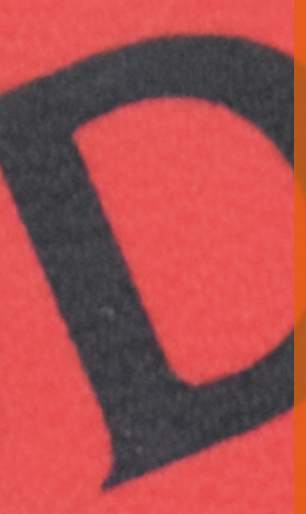

3.2 Textes relatifs à la protection du milieu aquatique

- Directive 2000/60/CE cadre sur l'eau (DCE)

- Directive 2008/56/CE sur la stratégie pour le milieu marin (DCSMM)

- Directives filles de la directive DCE et autres directives sur l'eau

3.3 Autres textes

- Directive 2010/75/UE sur les émissions industrielles (ex. directive IPPC de 2008)

- Directive Seveso 96/82/CE sur la maîtrise des dangers liés aux accidents majeurs impliquant des substances dangereuses modifiée et abrogée par la directive 2012/18/UE

- Règlement 2007/1907/CE sur les substances chimiques REACH

\section{RÉGLEMENTATION FRANÇAISE}

4.1 Réglementation dans le domaine nucléaire

- Titre IX du livre V du Code de l'environnement (partie législative issue de la codification de la loi $n^{\circ}$ 2006-686 dite TSN et réglementaire issue de la codification du décret $n^{\circ}$ 2007-15557 dit Procédures)

- Arrêté du 7 février 2012 fixant les règles générales relatives aux installations nucléaires de base modifié (INB)

4.2 Réglementation des installations classées pour

la protection de l'environnement (« ICPE »)

- Titre $1^{\text {er }}$ du livre V du Code de l'environnement (partie législative et réglementaire)

4.3 Textes sur l'eau et les milieux aquatiques

- Titre $1^{\text {er }}$ du livre II du Code de l'environnement (partie législative et réglementaire)

4.4 Textes sur l'air

- Titre II du livre II du Code de l'environnement (partie législative et réglementaire)

\section{TEXTES PROPRES À CHAQUE CENTRALE NUCLÉAIRE D'EDF}

5.1 Articulation des principaux textes conduisant aux autorisations

de prélèvements d'eau et de rejets

5.2 Textes propres à chaque centrale nucléaire d'EDF

- Prélèvements d'eau et rejets selon la loi TSN codifiée (DAC, décisions de I'ASN, RGE)

- Prélèvements d'eau et rejets d'effluents selon de décret du 4 mai 1995

- Autres prescriptions applicables

- Occupation du domaine public fluvial et maritime

Pour en savoir plus

Annexe 5.1 : Tableau récapitulatif des principaux textes réglementaires

Annexe 5.2 : Nature des différents types de textes législatifs et réglementaires

Annexe 5.3 : Code de l'environnement

Annexe 5.4 : Institutions de l'Union européenne 


\section{Cadre général}

La réglementation relative à la protection de l'environnement est composée de règles juridiques (dispositions) portant notamment sur :

- les éléments de l'environnement : air, eau, sols, écosystèmes ;

- les activités humaines telles que celles des installations nucléaires de base (INB) - dont font partie les centrales nucléaires - ou des installations classées pour la protection de l'environnement (ICPE) ;

- des sujets particuliers : fabrication et usage de substances chimiques, gestion des déchets.

Ces règles (normes) tendent à devenir de plus en plus techniques et complexes au fur et à mesure des avancées scientifiques et techniques. Elles sont déclinées dans des systèmes juridiques hiérarchisés en droit international, communautaire, national, voire local. Outre leur caractère juridiquement contraignant (obligations), ces règles de droit comportent également des recommandations aux différents acteurs.
Les principales dispositions législatives et réglementaires applicables dans le domaine de l'environnement ont été codifiées dans le Code de l'environnement (cf. annexe 5.3). Celui-ci intègre les règles établies notamment au regard du droit international (traités, conventions, protocoles) et du droit communautaire, dont la transposition est la source de plus de $85 \%$ du droit français de l'environnement. Depuis le décret du 14 mars 2019, une partie significative des textes relatifs aux INB sont intégrés au Code de l'environnement (codifiés).

Les prélèvements d'eau et les rejets d'effluents des centrales nucléaires d'EDF nécessitent l'obtention d'autorisations délivrées par les autorités compétentes françaises dans le cadre général de cette réglementation (cf. § 5).

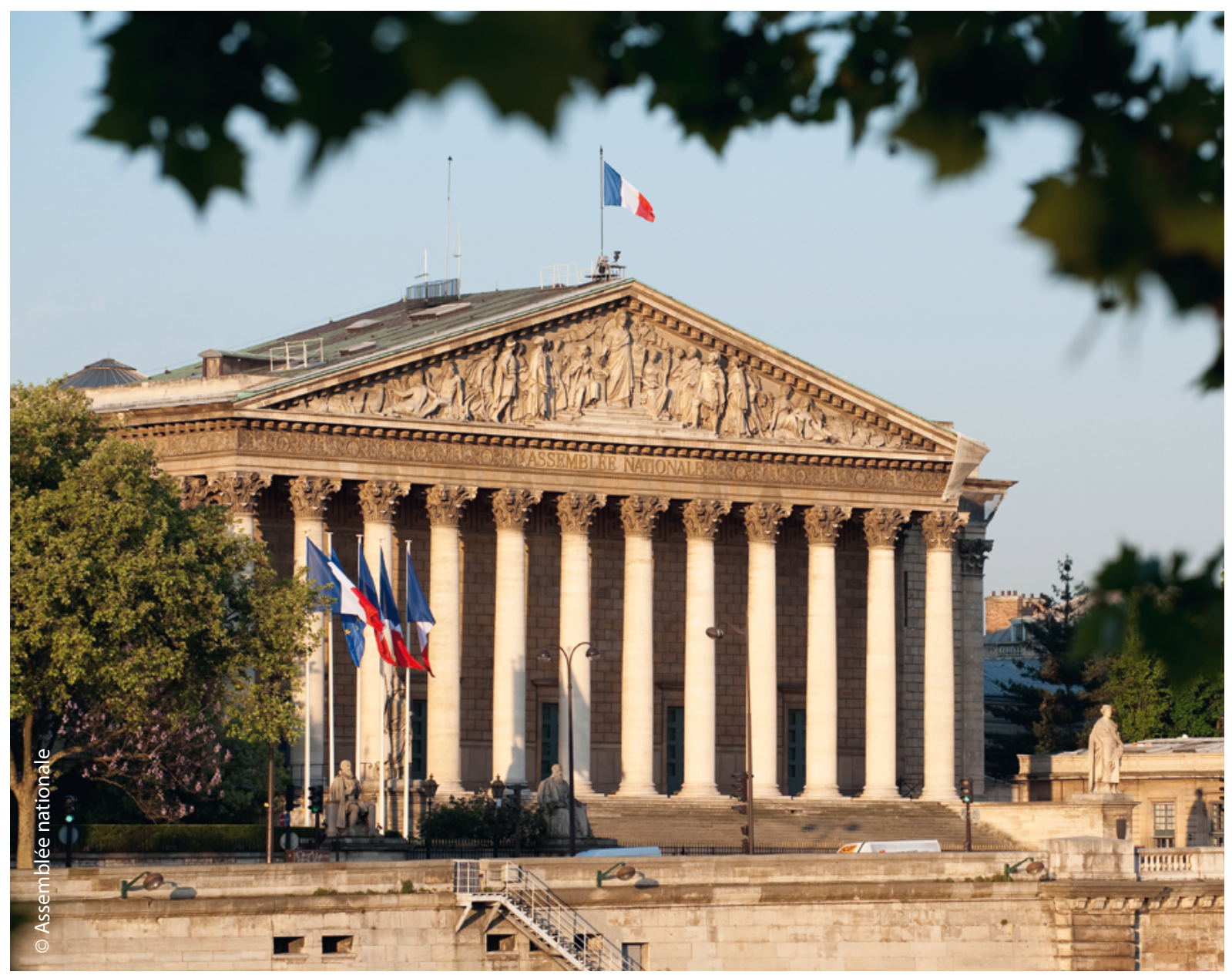




\section{Code de l'environnement - Charte de l'environnement Principes généraux sous-tendant la réglementation}

\section{Code de l'environnement}

L'article L. 110-1 du Code de l'environnement dispose que « les espaces, ressources et milieux naturels [...], font partie du patrimoine commun de la nation.[...] leur protection [...], sont d'intérêt général et concourent à l'objectif de développement durable qui vise à satisfaire les besoins de développement et la santé des générations présentes sans compromettre la capacité des générations futures à répondre aux leurs. »

Ce même article énonce également que sa protection, sa mise en valeur, et sa remise en état s'inspirent des principes généraux sur lesquels repose le droit de l'environnement, que l'on retrouve également sous une forme parfois différente dans la Charte de l'environnement promulguée en $2005^{7}$ et adossée à la Constitution, à savoir :

- le principe de précaution (article 5 de la Charte de l'environnement), selon lequel l'absence de certitudes, compte tenu des connaissances scientifiques et techniques du moment, ne doit pas retarder l'adoption de mesures effectives et proportionnées visant à prévenir un risque de dommages graves et irréversibles à l'environnement à un coût économiquement acceptable ;

- le principe d'action préventive et de correction, par priorité à la source (article 3 de la Charte de l'environnement), des atteintes à l'environnement, en utilisant les meilleures techniques disponibles à un coût économiquement acceptable (MTD) ;

- le principe pollueur-payeur (article 4 de la Charte de l'environnement), selon lequel les frais résultant des mesures de prévention, de réduction de la pollution et de lutte contre celle-ci doivent être supportés par le pollueur;

- le principe de participation (article 7 de la Charte de l'environnement), selon lequel chacun a accès aux informations relatives à l'environnement, y compris celles relatives aux substances et activités dangereuses, et le public est associé au processus d'élaboration des projets ayant une incidence importante sur l'environnement ou l'aménagement du territoire (cf. chapitre 4 sur l'information du public).

\section{Charte de l'environnement}

La Charte de l'environnement, promulguée le $1^{\text {er }}$ mars 2005, énonce en termes généraux que « chacun a le droit de vivre dans un environnement équilibré et respectueux de la santé » (article 1 1 r) ainsi que « le droit d'accéder à l'information détenue par les autorités publiques et le droit de participer à l'élaboration des décisions publiques ayant une incidence sur l'environnement » (article 7). L'élévation au rang constitutionnel de cette Charte donne une assise plus forte à certains instruments et principes nécessaires à la politique publique dans le domaine de l'environnement (précaution, prévention, responsabilité).

Dans une décision de QPC (2019-823) du 31 janvier 2020, le Conseil constitutionnel a reconnu pour la première fois que «la protection de l'environnement » pouvait justifier des « atteintes à la liberté d'entreprendre ». II a ainsi fait produire, pour la première fois, des effets juridiques contraignants à la Charte.

1. La Charte de l'environnement a été adoptée le 24 juin 2004 et promulguée le 1er mars 2005 par la loi constitutionnelle nº 2005-205.

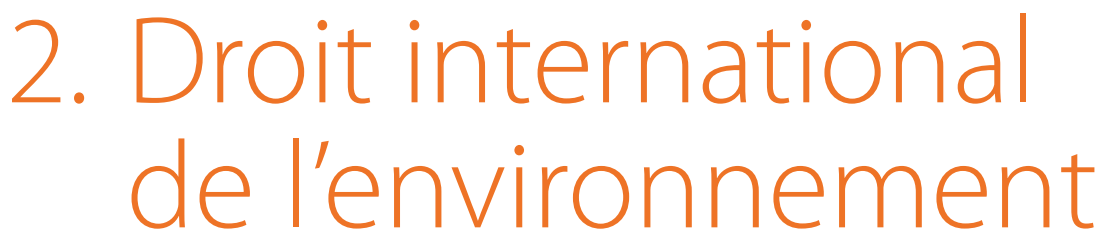

\subsection{ORGANISMES INTERNATIONAUX ET ORGANISATIONS NON GOUVERNEMENTALES CONCERNÉS}

Le droit international de l'environnement s'est développé à partir de la deuxième moitié du XXe siècle. Des centaines de textes internationaux ont vu le jour tant pour préserver les éléments de la biosphère (sols, eaux de surface et eaux souterraines continentales, océans, atmosphère), la biodiversité et la santé humaine - que pour tenter de résoudre les problèmes affectant les écosystèmes du fait des activités humaines.

La concertation entre les États est réalisée au travers de différents organismes internationaux créés, en général, sous l'égide des Nations unies (ONU). Ces organismes ou commissions élaborent des textes (accord, traité, 
convention, protocole, charte...) que les États volontaires (ou un groupement d'États comme l'Union européenne) signent puis ratifient. La ratification est lacte par lequel le signataire exprime de façon définitive son consentement à être lié par le texte international et à l'appliquer. Cette volonté se traduit également en droit communautaire (par l'adoption de règlements, directives) et en droit français dans des textes législatifs et réglementaires (par l'intervention de lois, décrets...).

Les organisations non gouvernementales (ONG), qui ne relèvent ni d'un État ni d'une institution internationale, sont devenues des acteurs incontournables des relations internationales. Elles interviennent dans la préparation et l'élaboration du droit de l'environnement et jouent un rôle d'alerte vis-à-vis du public et des décideurs. Elles participent aux débats et négociations des politiques de protection de l'environnement et assurent une surveillance informelle de l'application des obligations environnementales qui en découlent.

\subsection{PRINCIPAUX TEXTES INTERNATIONAUX RATIFIÉS PAR LA FRANCE}

\section{Protocole de Montréal relatif à des substances qui appauvrissent la couche d'ozone, $1987^{1}$}

Certaines substances chimiques émises dans l'atmosphère par les activités humaines appauvrissent la couche d'ozone stratosphérique (couche située entre 10 et $50 \mathrm{~km}$ de la surface de la Terre) dont le rôle est de filtrer les rayons ultraviolets du soleil qui sont nocifs pour la santé humaine et les écosystèmes. Le 16 septembre 2009, la Convention de Vienne et le Protocole de Montréal sont devenus les premiers traités dans l'histoire des Nations unies à atteindre la ratification universelle.

Ce protocole impose la suppression de l'utilisation des chlorofluorocarbones (CFC) sauf pour des utilisations qualifiées de critiques ou essentielles ${ }^{2}$, des halons, du bromure de méthyle et d'autres substances telles que les hydrochlorofluorocarbones (HCFC), le tétrachlorure de carbone, le bromochlorométhane, le hydrobromofluorocarbone et le méthylchloroforme.

Les centrales nucléaires sont concernées par ces dispositions car les matériels frigorifiques et les systèmes de climatisation utilisent certaines des substances visées. Un bilan des pertes de ces fluides doit être réalisé chaque année par l'exploitant.

\section{Conférence des Nations unies sur l'environnement et le développement (déclaration de Rio), 1992}

Au cours du sommet de la terre de Rio de 1992, les États membres de l'ONU ont adopté un texte fondateur du droit de l'environnement, comprenant 27 principes, intitulé « Déclaration de Rio sur l'environnement et le développement». Ce texte met au cœur de ces principes la notion de développement durable qui souligne que la protection de l'environnement doit faire partie intégrante du processus de développement et ne peut être considéré isolément. D'autres principes sont explicités à l'instar de celui du pollueur-payeur.

Outre la déclaration et ces principes, la Conférence permet l'adoption du programme Action 21 (Agenda 21 en anglais), qui énumère 2500 recommandations pour la mise en œuvre de la déclaration. La Convention sur le Climat a également été adoptée à cette occasion, et a abouti à la signature en 1997 du protocole de Kyoto affirmant la nécessité de réduire les gaz à effet de serre et met en place des objectifs à cet effet.

\section{Protocole de Kyoto sur la réduction des gaz à effet de serre, $1997^{3}$}

Le protocole de Kyoto, élaboré en 1997 et entré en vigueur en 2005, propose un calendrier de réduction des émissions des six gaz à effet de serre qui sont considérés comme la cause principale du réchauffement climatique des cinquante dernières années : dioxyde de carbone $\left(\mathrm{CO}_{2}\right)$, méthane $\left(\mathrm{CH}_{4}\right)$, hydrofluorocarbone (HFC), perfluorocarbone (PFC), hexafluorure de soufre $\left(\mathrm{SF}_{6}\right)$, peroxyde d'azote $\left(\mathrm{N}_{2} \mathrm{O}\right)$.

La France, qui a signé le Protocole en 1998, a entériné celui-ci en droit national au travers du décret $n^{\circ}$ 2005-295 du 22 mars 2005 portant publication du Protocole de Kyoto.

\section{Accord de Paris sur le climat, $2015^{4}$}

Dans le cadre de la $21^{e}$ Conférence des Parties à la Convention-cadre des Nations unies sur les changements climatiques (COP 21), a été signé l'Accord de Paris sur le climat, premier accord universel sur la question du réchauffement climatique. Au cœur de l'accord, sont inscrits les objectifs de maintenir en dessous de $2{ }^{\circ} \mathrm{C}$ la montée de la température globale, de se désinvestir des énergies fossiles, et d'atteindre la neutralité carbone. De même, le principe de responsabilités communes mais différenciées établi en 1992 au Sommet de Rio est rappelé, différenciant les pays les plus industrialisés et les pays en voie de développement, ainsi qu'un plancher d'aide climatique à ces derniers de 100 milliards d'euros par année.

Les objectifs de l'Accord doivent être révisés chaque année au niveau national jusqu'à 2020, puis tous les cing ans, pour pouvoir établir un premier bilan de l'Accord en 2023. 


\section{Quelques organismes internationaux et organisations non gouvernementales concernés par l'environnement et le secteur nucléaire}

\section{Organismes internationaux}

Certains organismes internationaux ont un rôle important dans l'élaboration de la réglementation dans le domaine de l'environnement et de la santé du public. Ils désignent les organisations intergouvernementales émanant des pouvoirs publics. Ils sont à distinguer des ONG qui émanent des membres privés de différents pays.

L'Agence internationale de l'énergie atomique (AIEA), créée en 1956, dont le siège est à Vienne (Autriche), fait partie des six organismes autonomes des Nations unies, avec entre autres I'Organisation mondiale de la santé (OMS). Dans le domaine de la radioprotection notamment, l'AIEA produit des documents qui servent de référence dans le monde entier : certains d'entre eux sont intégrés directement dans les réglementations nationales ou pris en compte par la Commission européenne pour préparer des directives.

L'Agence pour l'énergie nucléaire (AEN), est une agence spécialisée de l'OCDE, créée en 1958, et dont le but est d'aider ses pays membres à maintenir une utilisation sûre de l'énergie nucléaire à des fins pacifiques. Elle s'emploie pour cela à fournir des évaluations et dégager des convergences de vues.

La Communauté européenne de l'énergie atomique (EURATOM), créée en 1958, est un organisme public européen chargé de coordonner les programmes de recherche sur l'énergie nucléaire. Son but était en 1958 notamment la formation et la croissance rapide des industries nucléaires, en renforçant la recherche, facilitant les investissements et en assurant des débouchés à l'industrie nucléaire.

Le Programme des Nations unies pour l'environnement (PNUE), créé en 1972 lors du Sommet de la Terre de Stockholm, est un organe statutairement subsidiaire au Conseil économique et social. Basé à Nairobi au Kenya, le PNUE est la voix de I'ONU pour l'environnement. II coordonne les politiques de cette dernière en matière d'environnement. II assiste également les différents pays dans la mise en œuvre de leurs politiques environnementales, et a un rôle de promotion du développement durable.

La Commission internationale de protection radiologique (CIPR), créée en 1928, est une organisation internationale indépendante et émet des recommandations en matière de radioprotection. Ses recommandations n'ont pas force de loi, mais, de fait, sont reprises dans les réglementations nationales, soit directement, soit, dans le cas de l'Union européenne, après transposition sous forme de directives.

Le Comité scientifique des Nations unies sur l'effet des radiations atomiques (UNSCEAR - United Nations Scientific Committee on the Effects of Atomic Radiations), créé en 1955 est le comité des Nations unies qui a pour mission l'étude des effets biologiques des rayonnements ionisants. Sa vocation est exclusivement scientifique. Il établit notamment une synthèse des expositions moyennes dues aux différentes sources d'exposition.

\section{Organisations non gouvernementales (ONG)}

L'Association des régulateurs nucléaires de l'Europe de l'Ouest (WENRA - Western European Nuclear Regulators Association), créée en 1999, dont le but était à l'origine de développer une approche commune quant à la sûreté nucléaire ainsi qu'une capacité d'analyse indépendante de ces attendus, a évolué pour permettre aux régulateurs d'échanger des connaissances et discuter des questions de sûreté majeures.

L'Association mondiale des exploitants nucléaires (WANO - World Association of Nuclear Operators), créée en 1989, promeut la diffusion de l'information entre les exploitants nucléaires, et organise des revues de pairs sur les sites des différents membres afin de diffuser les meilleures pratiques.

L'Union internationale pour la conservation de la nature (UICN), fondée en 1948, est basée en Suisse et est la plus importante ONG en charge de la conservation de la nature en termes d'intégrité et de diversité des ressources naturelles. L'UICN regroupe des États, des agences gouvernementales et des organisations non gouvernementales. Elle est accréditée auprès de l'ONU. Sa mission est d'influencer, d'encourager et d'assister les sociétés dans le monde entier, dans la conservation de l'intégrité de la nature et de la biodiversité, ainsi que de s'assurer que l'utilisation de ces ressources naturelles est faite de façon équitable et durable. Sa liste rouge des espèces menacées est une référence mondiale.

Le Fonds mondial pour la nature (WWF), créé en 1961, est une organisation non gouvernementale internationale de protection de la nature et de l'environnement, fortement impliqué dans la protection de la biodiversité et le développement durable.

De nombreuses ONG concernant les thématiques environnementale et nucléaire se sont créées au cours des dernières décennies, parmi lesquelles : France nature environnement (1968), Greenpeace (1971) ou encore le Réseau sortir du nucléaire (1997). 


\section{Thématique - Eau :}

\section{Convention de Barcelone sur la protection du milieu marin et du littoral méditerranéen (protocole d'Athènes), 1976}

La convention a été signée en 1976 à Barcelone, sous l'égide du Programme des Nations unies pour l'environnement (PNUE), pour protéger la mer Méditerranée contre la pollution. Ratifiée par la France en 2001, cette convention est entrée en vigueur en 2004.

Cette convention comporte plusieurs protocoles, dont le protocole sur la pollution d'origine terrestre dit Protocole d'Athènes. Il fixe pour objectif la prise de mesures pour prévenir, combattre et éliminer « dans toute la mesure du possible " la pollution en mer Méditerranée due aux déversements par les fleuves, les établissements côtiers ou les émissaires, et vise en particulier les rejets toxiques susceptibles de bio-accumulation; les substances visées sont énumérées dans une liste annexée au protocole (détergents non biodégradables, métaux lourds, biocides et leurs dérivés, substances radioactives si les rejets sont non conformes aux principes de radioprotection, microorganismes pathogènes, hydrocarbures) mais aussi les composés azotés ou phosphorés pouvant être la cause d'eutrophisation et les rejets thermiques.

\section{Convention sur la protection du milieu marin de l'Atlantique Nord-Est, dite OSPAR (contraction de OSlo-PARis), $1992^{2}$}

La protection des eaux marines de l'Atlantique NordEst contre la pollution d'origine terrestre (convention de Paris de juin 1974) et la pollution provenant des immersions en mer (convention d'Oslo de 1972) fait l'objet d'une convention unique dénommée OSPAR, qui est entrée en vigueur le 25 mars 1998 (cf. chapitre 3 et chapitre 11).

Cette convention revêt une grande importance pour toutes les installations dont les rejets (radioactifs ou non) aboutissent dans les mers nord-européennes définies par OSPAR (cf. figure 1). Toutes les centrales nucléaires d'EDF sont donc concernées à l'exception de celles situées sur le Rhône, visées par le protocole d'Athènes.

Au cours d'une conférence tenue à Sintra (Portugal) ${ }^{3}$ en 1998, les ministres de l'Environnement des 15 pays signataires ont adopté, le 24 juillet, une stratégie portant sur la réduction progressive des substances dangereuses en général, et sur d'autres thèmes visant à protéger l'environnement marin tels que la lutte contre l'eutrophisation.

Pour les substances chimiques dangereuses, cette déclaration prévoit la réduction des rejets, de façon à parvenir à des teneurs dans l'environnement proche
Fig. 1 Zones maritimes couvertes par la convention OSPAR.

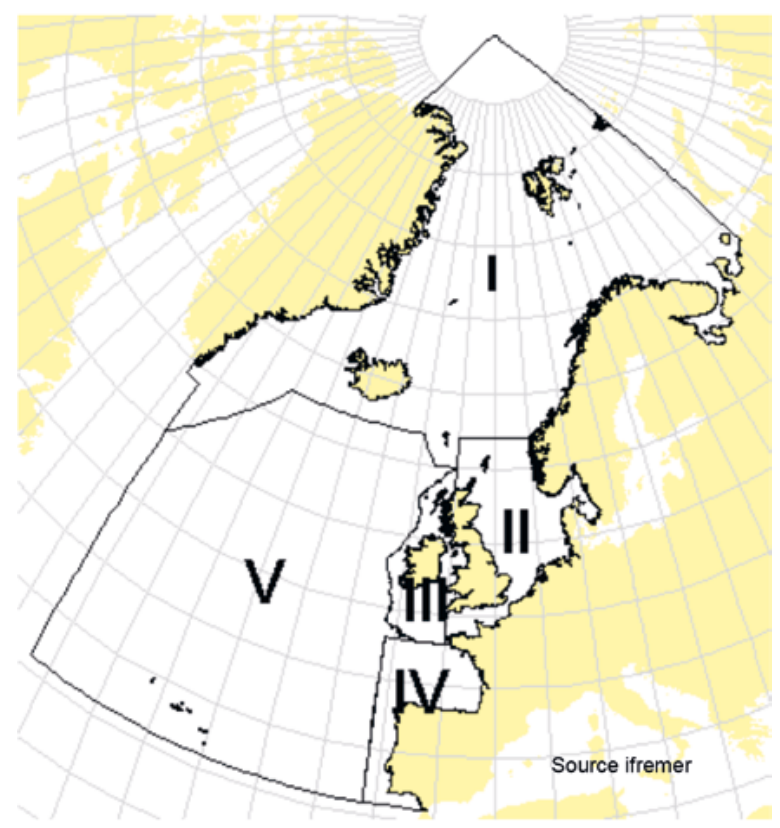

des teneurs ambiantes pour les substances présentes à l'état naturel, et proches de zéro pour les substances de synthèse. À cet objectif général fermement affiché est associée une volonté (" nous ferons tout notre possible pour progresser dans le sens de ») d'atteindre une cessation pure et simple des rejets d'ici l'an 2020.

Pour les substances radioactives, la convention fixe pour objectif "de parvenir d'ici 2020 à des teneurs, dans l'environnement, proches des teneurs ambiantes dans le cas des substances radioactives présentes à l'état naturel, et proches de zéro dans le cas des substances radioactives artificielles", ceci au moyen de "réductions progressives et substantielles des rejets, émissions ou pertes radioactives » (cf. chapitre 11 §5).

\section{Convention de Berne pour la protection du Rhin, $1999^{4}$}

En signant une nouvelle Convention pour la Protection du Rhin le 12 avril 1999 à Berne, les Gouvernements des cinq États riverains du Rhin (Suisse, France, Allemagne, Luxembourg, Pays-Bas) et le représentant de la Commission européenne ont formellement souligné qu'il convenait de protéger le caractère précieux du Rhin, de ses berges et de son milieu alluvial en renforçant plus encore la coopération. Cette convention, entrée en vigueur en 2003, remplace l'ancienne convention de Berne de 1963.

Les travaux réalisés dans le cadre de cette Convention sont suivis par la Commission internationale pour la protection du Rhin, composée de représentants des États signataires.

1. Signée en 1976, entrée en vigueur en 2004.

2. Ouverte à la signature le 22 décembre 1992, entrée en vigueur le 25 mars 1998

3. La déclaration de SINTRA ne constitue toutefois ni une décision ni une recommandation au sens de la Convention; elle peut s'analyser comme un simple engagement de nature politique.

4. Signée le 12 avril 1998, entrée en vigueur en 2003. 
Accord international de Gand sur la Meuse, $2002^{1}$

La Commission internationale de la Meuse a été créée en 2002 par la signature de l'accord international sur la Meuse. L'objectif de l'accord est la gestion durable et globale de l'eau du district hydrographique de la Meuse. L'accord a été signé par la Région wallonne, les Pays-Bas, la France, l'Allemagne, la Région flamande, la Région de Bruxelles-Capitale, la Belgique et le Luxembourg et il est entré en vigueur en 2005 (cf. \& 5 à propos des centrales frontalières : centrale de Chooz).

\section{Thématique - Participation du Public \& Information environnementale : \\ Convention d'Aarhus sur l'accès à l'information, la participation du public au processus décisionnel et l'accès à la justice en matière d'environnement, $1998^{2}$}

La Convention d'Aarhus a trois objectifs majeurs : améliorer l'information environnementale délivrée par les autorités publiques, vis-à-vis des principales données environnementales, favoriser la participation du public à la prise de décisions ayant des incidences sur l'environnement, et étendre les conditions d'accès à la justice en matière de législation environnementale et d'accès à l'information.

Ces principes ont été repris en France par la Charte de l'environnement de 2005 qui reconnaît le droit de chacun à l'accès aux informations relatives à l'envi- ronnement et la participation aux décisions publiques ayant une incidence sur l'environnement.

L'accès à la justice n'est toutefois pas inscrit dans la Charte, mais le Tribunal de l'Union européenne a pu reconnaître que les ONG avaient notamment accès à la justice en matière d'environnement du fait de la Convention d'Aarhus ${ }^{3}$.

\section{Convention d'Espoo relative à l'évaluation de l'impact sur l'environnement dans un contexte transfrontière, $1991^{4}$}

La Convention d'Espoo a pour objet de s'assurer que les États, parties à la Convention, prennent toutes les mesures appropriées et efficaces pour prévenir, réduire et maîtriser l' "impact transfrontière préjudiciable important » que des activités ou projets (parmi lesquels figurent les centrales nucléaires) pourraient avoir sur l'environnement. Plus concrètement, deux obligations en sont issues:

- réaliser l'évaluation de l'impact sur l'environnement de certaines activités et projets ;

- mener les procédures de notification et de consultation des autres états parties sur ces activités et projets.

La Convention a, par ailleurs, fait l'objet de deux amendements : l'amendement de Sofia et l'amendement de Cavtat ${ }^{5}$. La Convention d'Espoo a été complétée par le Protocole de Kiev adopté le 21 mai 2003. Le protocole est entré en vigueur le 11 juillet 2010.

\section{Réglementation européenne}

L'environnement fait partie des domaines dans lesquels les pays de l'Union européenne ont reconnu, dès les années 1970, l'intérêt d'une approche commune. Cette approche se caractérise par deux types d'actions :

- la détermination de règles communes pour lutter contre les pollutions globales pouvant avoir un impact sur l'environnement et sur l'Homme qui en fait partie ;

- la fixation de normes communes concernant notamment la qualité de l'eau, l'exposition du public aux rayonnements ionisants (radioprotection).

L'Union européenne entend aujourd'hui prendre une place centrale et grandissante dans l'impulsion des politiques mondiales de protection de l'environnement: en témoigne le traité de Lisbonne du
13 décembre 2007 qui intègre la lutte contre le changement climatique dans ses objectifs, la création en 2010 d'un commissaire à l'action pour le climat, et l'adoption le 22 janvier 2014 du cadre pour le climat et l'énergie à l'horizon 2030 qui prévoit des objectifs particulièrement ambitieux (réduire les émissions de gaz à effet de serre d'au moins 40 \% (par rapport aux niveaux de 1990), porter la part des énergies renouvelables à au moins $27 \%$, améliorer l'efficacité énergétique d'au moins $27 \%$.).

La réglementation européenne, dans le domaine de l'environnement et de la santé du public, dérive souvent de textes internationaux dont l'Union européenne est partie prenante. Elle conditionne une grande partie de la réglementation nationale des pays membres.

1. Signé le 3 décembre 2002, entré en vigueur en 2006.

2. Signée le 25 juin 1998, entrée en vigueur en 2001.

3. Décision Tribunal de I'Union européenne, Stichting Natuur en Milieu c./Commission européenne, T-338/08, 14 juin 2012.

4. Adoptée le 25 février 1991 par la Commission économique pour l'Europe des Nations unies (CEE-ONU), puis entrée en vigueur le 10 septembre 1997.

5. Le premier est porté par la décision II/14 adoptée à Sofia le 27 février 2001 qui est entrée en vigueur le 26 août 2014. Le second est porté par la décision III/7 adoptée à Cavtat le 4 juin 2004, entré en vigueur le 23 octobre 2017. 
Parmi les différents instruments juridiques communautaires (cf. annexe 5.2), la directive européenne est l'acte normatif qui domine en matière d'environnement. Celle-ci doit être transposée dans le droit national pour pouvoir produire ses effets. Si ses termes sont suffisamment clairs et précis, la directive peut s'appliquer directement dans le droit national, une fois le délai de transposition échu.

Certaines dispositions législatives et réglementaires appliquées aux installations nucléaires de base (INB) et aux centrales nucléaires sont tirées du droit communautaire, il est donc nécessaire d'en présenter, ici, les éléments marquants.

\subsection{TEXTES RELATIFS À LA PROTECTION DE LA SANTÉ PUBLIQUE}

\section{Le Traité EURATOM}

Ce traité a été signé en $1957^{2}$ dans le but de permettre le développement de l'énergie nucléaire tout en assurant la protection de la population et des travailleurs contre les effets nocifs des rayonnements ionisants. Ce traité comporte plusieurs articles qui concernent la protection du public contre les effets des rayonnements ionisants (Art. 30 à 33), les rejets radioactifs et la radioactivité dans l'environnement (Art. 35 à 38). Parmi ces articles, l'article 37 intéresse particulièrement l'exploitant lorsque celui-ci envisage de construire une installation nouvelle ou de modifier une installation existante pouvant entraîner une augmentation des rejets radioactifs (cf. encart) ${ }^{3}$.

\section{Directive 2013/59/Euratom du Conseil du 5 décembre 2013 fixant les normes de base relatives à la protection sanitaire contre les dangers résultant de l'exposition aux rayonnements ionisants et abrogeant les directives 89/618/Euratom, 90/641/Euratom, 96/29/Euratom, 97/43/Euratom et 2003/122/Euratom}

La directive a pour objet, d'une part, de fusionner cinq directives relatives à la radioprotection et, d'autre part, de prendre en compte les données scientifiques les plus récentes dans le domaine. Elle ne remet pas en cause les fondamentaux de la directive 96/29/ Euratom du 13 mai 1996, qu'elle a abrogé, à savoir :

- le principe de justification : l'activité humaine impliquant une exposition aux rayonnements ionisants doit pouvoir être justifiée par les avantages qu'elle procure, après avoir pris en compte l'ensemble des avantages et des inconvénients ;

- le principe de l'optimisation de la protection : non seulement les expositions doivent être justifiées mais, de plus, elles doivent être maintenues à un niveau aussi bas qu'il est raisonnablement possible de le faire ; le principe d'optimisation est également

\section{Articles clés du Traité EURATOM}

L'Article 35 : Chaque État membre établit les installations nécessaires pour effectuer le contrôle permanent du taux de la radioactivité de l'atmosphère, des eaux et du sol ainsi que le contrôle du respect des normes de base. La Commission a le droit d'accéder à ces installations de contrôle ; elle peut en vérifier le fonctionnement et l'efficacité.

L'article 36 : Les renseignements concernant les contrôles visés à l'article 35 sont communiqués régulièrement par les autorités compétentes à la Commission, afin que celle-ci soit tenue au courant du taux de la radioactivité susceptible d'exercer une influence sur la population.

L'article 37 : Chaque État membre est tenu de fournir à la Commission les données générales de tout projet de rejet d'effluents radioactifs sous n'importe quelle forme, permettant de déterminer si la mise en œuvre de ce projet est susceptible d'entraîner une contamination radioactive des eaux, du sol ou de l'espace aérien d'un autre État membre. La Commission, après consultation du groupe d'experts visé à l'article 31, émet son avis dans un délai de six mois.

L'article 38 : La Commission adresse aux États membres toute recommandation en ce qui concerne le taux de radioactivité de l'atmosphère, des eaux et du sol. En cas d'urgence, la Commission arrête une directive par laquelle elle enjoint à l'État membre en cause de prendre, dans le délai qu'elle détermine, toutes les mesures nécessaires pour éviter un dépassement des normes de base et pour assurer le respect des réglementations. Si cet État ne se conforme pas, dans le délai imparti, à la directive de la Commission, celle-ci ou tout État membre intéressé peut, par dérogation aux articles 258 et 259 du traité sur le fonctionnement de l'Union européenne, saisir immédiatement la Cour de justice de l'Union européenne.

appelé « principe ALARA » (As Low As Reasonably Achievable);

- le respect de limites ou de niveaux de dose à ne pas dépasser (principe de limitation) : ces limites ou niveaux dépendent des circonstances, ainsi elles sont plus sévères pour les enfants, et les femmes enceintes et allaitantes.

En effet, le système de la radioprotection fixé par la directive 2013/59 continue de reposer sur ces trois piliers dont les définitions ont été précisées.

Sur le fondement du principe d'optimisation, la directive donne un rôle plus important aux contraintes de dose qui s'appliquent désormais explicitement aux doses équivalentes et non plus seulement à la dose 
efficace. Les limites de doses n'ont pas été modifiées, excepté pour le cristallin. La limite de dose efficace pour l'exposition de la population à des rayonnements ionisants est fixée à $1 \mathrm{mSv}$ par an (et la limite de dose équivalente est fixée à $15 \mathrm{mSv}$ par an pour le cristallin $)^{1}$

Le contrôle réglementaire est désormais fondé sur une approche graduée, en ce qu'il prévoit en fonction des risques induits, une notification ou une autorisation, celle-ci pouvant prendre la forme d'un enregistrement ou d'une licence.

La directive étend les modalités de protection des travailleurs extérieurs intervenant en zones contrôlées prévues par la directive 90/641/Euratom aux travailleurs intervenant dans les zones surveillées.

La directive prévoit désormais deux types de fonction dans le domaine de la radioprotection l'« expert en radioprotection ", et la "personne chargée de la radioprotection » (dans l'ancienne directive, ces deux fonctions se confondaient dans la fonction d'« expert qualifié »).

En ce qui concerne les situations d'exposition d'urgence, l'objet de la directive est de mettre en place un système plus complet comprenant une évaluation des situations d'exposition d'urgence potentielles, un système global de gestion des urgences, des plans d'urgence et des stratégies préplanifiées pour la gestion de chaque événement.

Les mesures relatives à l'exposition aux matières radioactives naturelles (radon et aux matériaux de construction) ne sont plus limitées aux seules expositions professionnelles, mais sont désormais étendues à l'exposition du public.

La transposition de la directive 2013/59 a principalement été réalisée par l'adoption des décrets n²018437 du 4 juin 2018 relatif à la protection des travailleurs contre les risques dus aux rayonnements ionisants ; et $n^{\circ}$ 2018-438 du 4 juin 2018 relatif à la protection contre les risques dus aux rayonnements ionisants auxquels sont soumis certains travailleurs qui ont respectivement modifié le Code du travail et le Code de la santé publique ainsi que par l'introduction dans le Code de l'environnement, aux articles R. 593-112 à R. 593-114, de dispositions relatives au " conseiller en radioprotection ». Ces dispositions prévoient dans les INB, la mise en place de "pôle de compétence ».

\section{Décision $n^{\circ}$ 1082/2013/UE relative aux menaces transfrontières graves sur la santé}

La décision établit les règles sur la surveillance des menaces transfrontières graves pour la santé, vise à appuyer la coopération et la coordination entre les pays de l'UE pour lutter contre ces menaces transfrontières, et précise les modalités de la coopération et de la coordination entre les différents acteurs au niveau de l'UE.

\section{Directive européenne nº 98/83/CE du 03 novembre 1998 relative à la qualité des eaux destinées à la consommation humaine}

La directive vise à protéger la santé des personnes en établissant des exigences de salubrité et de propreté auxquelles doit satisfaire l'eau potable dans la Communauté. Elle s'applique à toutes les eaux destinées à la consommation humaine, à l'exception des eaux minérales naturelles et des eaux médicinales.

Ce texte a été transposé en droit français par le décret n²001-1220 du 20 décembre 2001 relatif aux eaux destinées à la consommation humaine, à l'exclusion des eaux minérales naturelles, codifié dans le Code de la santé publique.

La directive introduit pour la première fois des paramètres de qualité relatifs à la radioactivité. L'un porte sur le tritium (100 Bq/ $\left.\mathrm{L}^{2}\right)$, l'autre sur la dose reçue $\mathrm{du}$ fait de l'ingestion d'eau (0,1 mSv/an). Ces valeurs ne sont pas des limites mais simplement des paramètres indicateurs. En cas de dépassement, les États membres sont tenus d'informer la Commission européenne et la population, de rechercher la source de la contamination et, éventuellement, de prendre des mesures pour réduire les niveaux d'activité en tritium dans l'eau potable.

La dose indicative totale (0,1 mSv/an) ne concerne pas uniquement le tritium mais l'ensemble des radioéléments à l'exclusion du potassium 40 et du radon et ses descendants.

Cette directive a été modifiée par la directive n²015/1787/UE du 06 octobre 2015 concernant certaines exigences minimales des programmes de contrôle pour toutes les eaux destinées à la consommation humaine et les spécifications pour la méthode d'analyse de différents paramètres. Toutefois, la directive 2013/51/Euratom ${ }^{3}$ ayant introduit des dispositions spécifiques pour le contrôle des substances radioactives, les programmes de contrôle desdites substances ont donc été exclues de la directive 2015/1787.

\section{Directive européenne 2006/7/CE du 15 février 2006 concernant la gestion de la qualité des eaux de baignade et abrogeant la directive 76/160/CEE}

Cette directive vise à préserver, protéger et à améliorer la qualité de l'environnement ainsi qu'à protéger la santé humaine, en complément de la directive 2000/60 (ci-après). Elle s'applique à toute partie des

1. Confer en ce sens l'article R. 1333-11 du Code de la santé publique.

2. Nota : L'OMS a fixé une valeur guide pour le tritium de $10000 \mathrm{~Bq} / \mathrm{L}$. Cette valeur a été déterminée en considérant qu'une consommation quotidienne de 2 litres d'eau potable à 10000 Bq/L n'entraîne pas de dose supérieure à 0,1 mSv, soit 1/10e de la limite réglementaire fixée pour le public: $1 \mathrm{mSv} / \mathrm{an}$. 3. Directive 2013/51/Euratom du Conseil du 22 octobre 2013 fixant des exigences pour la protection de la santé de la population en ce qui concerne les substances radioactives dans les eaux destinées à la consommation humaine. 
eaux de surface où l'autorité compétente de l'État membre s'attend à ce qu'un grand nombre de personnes se baignent. Cette directive a été transposée en droit français, notamment, par la loi dite " LEMA », codifiée dans le Code de l'environnement (cf. § 4.2).

\subsection{TEXTES RELATIFS À LA PROTECTION DU MILIEU AQUATIQUE}

\section{Directive 2000/60/CE du Parlement et du Conseil adoptée le 23 octobre 2000, établissant un cadre pour une politique communautaire dans le domaine de l'eau (directive dite "cadre sur l'eau», «DCE»)'}

Cette directive a été transposée, notamment, par la Loi dite «LEMA » (cf. § 4.2) C'est l'élément majeur de la réglementation européenne en matière de protection de la ressource en eau. Avec les directives relatives à la santé publique, la directive-cadre sur l'eau fait partie des textes communautaires ayant une grande importance pour les activités des centrales nucléaires s'agissant des prélèvements d'eau et des rejets d'effluents.

La DCE a été modifiée par la directive n²014/101/ UE du 30 octobre 2014 afin d'assurer la qualité et la comparabilité des méthodes utilisées pour le contrôle des paramètres types définis sous la responsabilité des États membres aux fins de la surveillance écologique des eaux.

La DCE est appelée à faire l'objet d'une refonte globale (prévue par la directive elle-même). En ce sens, la DCE et ses directives filles doivent faire l'objet d'un réexamen quant au fonctionnement de ses dispositions. Pour ce faire, une consultation de l'ensemble des citoyens européens s'est tenue ${ }^{2}$ sur la base de laquelle la Commission européenne a publié le cinquième rapport ${ }^{3}$ d'implémentation de la DCE et ses directives filles.

\section{Objectifs}

L'objectif de la politique communautaire dans le domaine de l'eau visait à atteindre, d'ici 2015, un « bon état » chimique et écologique des eaux superficielles et souterraines en Europe. Pour y parvenir, la directive prévoit d'instaurer une gestion de l'eau par bassin hydrographique, afin :

- d'améliorer la qualité des eaux et des écosystèmes ;

- de prévenir toute dégradation supplémentaire ;

- de promouvoir une utilisation durable de l'eau (usages) ;

- de réduire progressivement les émissions de substances chimiques dangereuses;

- de contribuer à atténuer les effets des inondations et des sécheresses;

- de protéger les eaux marines

- de réaliser les objectifs des accords internationaux.
Gestion de l'eau par bassin hydrographique Cette gestion par bassin hydrographique, qui s'inspire de la législation française (loi sur l'eau de 1964), est fondée sur :

- l'identification et l'analyse des masses d'eaux : les États membres sont tenus de recenser tous les bassins hydrographiques qui se trouvent sur leur territoire afin de les rattacher à des districts hydrographiques. Les bassins hydrographiques qui s'étendent sur le territoire de plus d'un État seront intégrés au sein d'un district hydrographique international. Chaque État membre désigne une autorité compétente pour chacun des districts.

Les États membres doivent faire une analyse des caractéristiques de chaque district hydrographique, une étude de l'incidence de l'activité humaine sur les eaux, une analyse économique de l'utilisation de celles-ci et un registre des zones qui nécessitent une protection spéciale;

- les mesures de protection et de restauration : un plan de gestion et un programme de mesures doivent être élaborés au sein de chaque district hydrographique. Ces mesures ont pour but de :

- prévenir la détérioration, améliorer et restaurer l'état des masses d'eau de surface afin d'atteindre un bon état chimique et écologique (cf. encart DCE) de celles-ci,

- réduire la pollution due aux rejets et émissions de substances chimiques dangereuses,

- protéger, améliorer et restaurer les eaux souterraines, prévenir leur pollution, leur détérioration et assurer un équilibre entre leurs captages et leur renouvellement,

- préserver les zones protégées tels que les sites Natura 2000 (cf. chapitres 3 et 8).

Les objectifs précédents devaient être atteints en 2015, mais cette échéance peut être rapportée ou assouplie, tout en respectant les conditions établies par la directive. Une liste de substances chimiques prioritaires présentant un risque important pour le milieu aquatique constitue l'annexe $X$ de la directive. Celle-ci a été modifiée par la directive-fille 2008/105/ CE sur les normes de qualité environnementales (NQE) et les zones de mélange (cf. encart DCE). Une détérioration temporaire des masses d'eau ne constitue pas une infraction de la présente directive si elle résulte des circonstances exceptionnelles et non prévisibles liées à un accident, une cause naturelle ou un cas de force majeure.

En France, les obligations de cette directive sont intégrées notamment dans les schémas directeurs d'aménagement et de gestion des eaux (SDAGE) et des schémas d'aménagement et de gestion des eaux (SAGE) élaborés et mis en œuvre au niveau de chaque bassin hydrographique (cf. §4.3).

1. Modifiée par la directive 2009/31/CE.

2. Septembre 2018 à mars 2019.

3. COM(2019)95 final. 


\section{Directive européenne cadre sur l'eau (DCE)}

Les grandes étapes de sa mise en œuvre par les états membres

- 2004 : État des lieux

- 2005 : Consultation du public sur l'état des lieux

- 2008 : Consultation du public sur les SDAGE

- 2009 : Publication du premier plan de gestion et du programme de mesures

- 2009 : Adoption des SDAGE révisés

- 2015 : Point sur l'atteinte des objectifs, suivi d'un second plan de gestion et programme de mesure

- 2027 : Dernière échéance pour la réalisation des objectifs

Mise en œuvre de la DCE vis-à-vis des objectifs de qualité d'eau

Centrée sur la préservation du milieu naturel et assortie d'une obligation de résultats, la DCE implique la planification et la mise en œuvre d'actions de diagnostic et, le cas échéant, de restauration des milieux aquatiques. La phase de diagnostic consiste à établir un état des lieux des milieux aquatiques. Au cours de cet état des lieux seront choisis des sites de référence qui permettront d'établir des valeurs de référence du " "bon état" chimique et écologique » pour les différents indicateurs de qualité chimique (concentrations de polluants) et biologique (invertébrés, diatomées, poissons, macrophytes...) pour chaque type de masse d'eau.

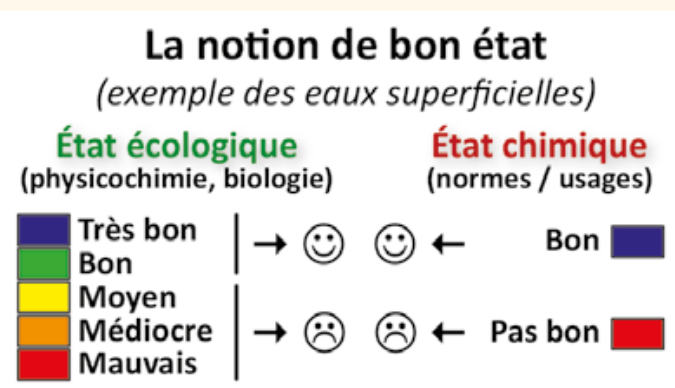

Une situation appréciée par rapport aux conditions de référence (très bon état)...

Termes-clés de la directive 2000/60/CE (art.2)

Bon état chimique d'une eau de surface : état d'une masse d'eau respectant les critères chimiques définis par l'annexe IX de la directive DCE ou d'autres textes communautaires pertinents fixant les normes de qualité environnementale.

Bon état écologique : état d'une masse d'eau respectant les critères biologiques de qualité définis par les textes communautaires, notamment l'annexe $V$ de la directive DCE.

Bon potentiel écologique : état d'une masse d'eau fortement modifiée hydromorphologiquement par l'homme ou artificielle conformes aux dispositions de l'annexe $\mathrm{V}$ de la directive DCE.

Bon état chimique d'une eau souterraine :état d'une masse d'eau répondant aux conditions de l'annexe V de la directive DCE. Norme de qualité environnementale (NQE) : la concentration d'un polluant ou d'un groupe de polluants dans l'eau, les sédiments ou le biotope qui ne doit pas être dépassée afin de protéger la santé humaine et l'environnement.

Masse d'eau de surface : il s'agit d'une partie distincte et significative des eaux de surface telles qu'un lac, un réservoir, une rivière, un fleuve ou un canal, une partie de rivière, de fleuve ou de canal, une eau de transition ou une portion d'eaux côtières.

Eaux intérieures : toutes les eaux stagnantes et les eaux courantes à la surface du sol et toutes les eaux souterraines en amont de la ligne de base servant pour la mesure de la largeur des eaux territoriales.

Eaux de surface : les eaux intérieures, à l'exception des eaux souterraines, les eaux de transition et les eaux côtières, sauf en ce qui concerne leur état chimique, pour lequel les eaux territoriales sont également incluses.

Eaux souterraines : toutes les eaux se trouvant sous la surface du sol dans la zone de saturation et en contact direct avec le sol ou le sous-sol.

Eaux de transition : des masses d'eaux de surface à proximité des embouchures de rivières, qui sont partiellement salines en raison de leur proximité d'eaux côtières, mais qui sont fondamentalement influencées par des courants d'eau douce.

Eaux côtières : les eaux de surface situées en-deçà d'une ligne dont tout point est situé à une distance d'un mille marin au-delà du point le plus proche de la ligne de base servant pour la mesure de la largeur des eaux territoriales et qui s'étendent, le cas échéant, jusqu'à la limite extérieure d'une eau de transition.

Bassin hydrographique : toute zone dans laquelle toutes les eaux de ruissellement convergent à travers un réseau de rivières, fleuves et éventuellement de lacs vers la mer, dans laquelle elles se déversent par une seule embouchure, estuaire ou delta.

District hydrographique : une zone terrestre et maritime, composée d'un ou plusieurs bassins hydrographiques ainsi que des eaux souterraines et eaux côtières associées, identifiée comme principale unité aux fins de la gestion des bassins hydrographiques.

Terme-clé de la directive-fille 2008/105/CE (art. 4) modifiée par la directive no 2013/39/UE

Zone de mélange : zone adjacente au point de rejet où les concentrations d'un ou plusieurs polluants peuvent dépasser les normes de qualité environnementales. L'arrêté du 25 janvier 2010 modifié relatif aux méthodes et critères d'évaluation de l'état écologique et chimique reprend cette définition en précisant que «Cette zone est proportionnée et limitée à la proximité du point de rejet et ne compromet pas le respect des NQE sur le reste de la masse d'eau ». 
Concertation avec les parties prenantes et aspects financiers

Chaque État membre doit encourager la concertation avec toutes les parties prenantes pour élaborer ces plans de gestion. Les États membres doivent assurer que la politique de tarification incite les consommateurs à utiliser les ressources de façon efficace et que les différents secteurs économiques contribuent à la récupération des coûts des services liés à l'utilisation de l'eau, y compris les coûts pour l'environnement et les ressources.

\section{Directive 2008/56/CE du 17 juin 2008 établissant un cadre d'action communautaire dans le domaine de la politique pour le milieu marin (directive-cadre «stratégie pour le milieu marin » (DCSMM)}

La directive ne porte sur le domaine littoral que de façon incomplète, puisque cet espace est déjà pris en compte partiellement par la directive-cadre sur l'eau (DCE). L'objectif principal de la Stratégie marine européenne est de parvenir à un bon état écologique du milieu marin dans l'Union européenne à l'horizon 2020.

\section{Directives « filles » de la directive DCE et autres directives relatives à l'eau}

La directive-cadre sur l'eau a donné naissance à des directives "filles » et d'autres textes relatifs à l'eau. Les principaux textes sont énumérés ci-après:

- directive 2006/44/CE du 6 septembre 2006 concernant la qualité des eaux douces ayant besoin d'être protégées ou améliorées pour être aptes à la vie des poissons ;

- directive 2006/118/CE du 12 décembre 2006 sur la protection des eaux souterraines contre la pollution et la détérioration. Cette Directive définit le cadre des dispositions à mettre en œuvre pour prévenir et réduire la pollution des eaux souterraines. Ceci passe, notamment, par des mesures d'évaluation de l'état chimique des eaux et des actions visant à réduire la présence de polluants ;

- directive 2007/60/CE du 23 octobre 2007 relative à l'évaluation et la gestion des inondations ;

- directive 2008/105/CE du 16 décembre 2008 établissant des normes de qualité environnementale dans le domaine de l'eau. Elle fixe des limites de concentration dans les eaux de surface pour 33 substances chimiques prioritaires et 8 autres polluants ;

- directive 2009/90/CE de la Commission du 31 juillet 2009 établissant, conformément à la directive 2000/60/CE du Parlement européen et du Conseil, des spécifications techniques pour l'analyse chimique et la surveillance de l'état des eaux; - directive 2014/80/UE de la Commission du 20 juin 2014 modifiant l'annexe II de la directive 2006/118/ CE du Parlement européen et du Conseil sur la protection des eaux souterraines contre la pollution et la détérioration? ;
- directive (UE) 2017/845 de la Commission du 17 mai 2017 modifiant la directive 2008/56/CE du Parlement européen et du Conseil en ce qui concerne les listes indicatives d'éléments à prendre en compte lors de la préparation des stratégies pour le milieu marin.

\subsection{AUTRES TEXTES}

Directive 2010/75/UE du 24 novembre 2010 relative aux émissions industrielles (dite IED » succédant à la directive 2008/1/CE du 15 janvier 2008 modifiée relative à la prévention et à la réduction intégrées de la pollution (dite "IPPC» : Integrated Pollution Prevention and Control qui est abrogée au 7 janvier 2014 par la directive IED)

Cette directive soumet notamment à autorisation les activités industrielles qui ont un fort potentiel de pollution. Elle vise à éviter ou à minimiser les émissions polluantes dans l'atmosphère, les eaux et les sols ainsi que les déchets provenant des installations industrielles en ayant recours aux meilleures techniques disponibles (MTD).

La notion de MTD définie par la directive IED est souvent évoquée lorsqu'il est question des prélèvements d'eau et des rejets d'effluents des centrales nucléaires

\section{Meilleures techniques disponibles (MTD)}

Extrait de la directive $2010 / 75 / U E$ : art. 3 point 10 : " "meilleures techniques disponibles": le stade de développement le plus efficace et avancé des activités et de leurs modes d'exploitation, démontrant l'aptitude pratique de techniques particulières à constituer, en principe, la base des valeurs limites d'émission visant à éviter et, lorsque cela s'avère impossible, à réduire de manière générale les émissions et l'impact sur l'environnement dans son ensemble.

a) Par "techniques", on entend aussi bien les techniques employées que la manière dont l'installation est conçue, construite, entretenue, exploitée et mise à l'arrêt ;

b) Par "disponibles", on entend les techniques mises au point sur une échelle permettant de les appliquer dans le contexte du secteur industriel concerné, dans des conditions économiquement et techniquement viables, en prenant en considération les coûts et les avantages, que ces techniques soient utilisées ou produites ou non sur le territoire de l'État membre intéressé, pour autant que l'exploitant concerné puisse y avoir accès dans des conditions raisonnables;

c) Par "meilleures", on entend les techniques les plus efficaces pour atteindre un niveau général élevé de protection de l'environnement dans son ensemble. »

1. Notamment transposé par l'arrêté du 17 décembre 2008 établissant les critères d'évaluation et les modalités de détermination de l'état des eaux souterraines et des tendances significatives et durables de dégradation de l'état chimique des eaux souterraines modifié. 
d'EDF. Certaines MTD sont décrites dans des documents de référence appelés BREF (Best available Technique References). Ces documents techniques sont élaborés par la Commission en concertation avec les parties prenantes (industriel, États, ONG...) et révisés périodiquement.

\section{Directives SEVESO 82/501/CE du 24 juin 1982, SEVESO II 96/82/CE du 9 décembre 1996 et 2003/105/CE du 16 décembre 2003 sur la maîtrise des dangers, et SEVESO III 2012/18/UE du 4 juillet 2012 concernant la maîtrise des dangers liés aux accidents majeurs impliquant des substances dangereuses}

La directive SEVESO de 1982 a permis d'initier le processus d'harmonisation des législations sur la maîtrise du risque. Modifiée en 1996, en 2003, et en 2012 la directive dite SEVESO constitue le pendant en matière de risques accidentels de la directive IED sur les pollutions. Parmi les changements les plus importants induits par la dernière réforme figurent des dispositions plus strictes concernant la participation du public au processus décisionnel et l'accès à la justice, concernant également les inspections des établissements, ainsi que des améliorations relatives à la manière dont les informations sont recueillies, gérées, mises à disposition.

\section{Règlement 1907/2006/CE du 18 décembre 2006} concernant l'enregistrement, l'évaluation et l'autorisation des substances chimiques, ainsi que les restrictions applicables à ces substances («REACH»)

Le règlement REACH (enRegistrement, Évaluation et Autorisation des substances $\mathrm{CH}$ Himiques) entré en vigueur

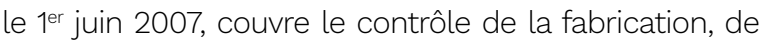
l'importation, de la mise sur le marché et de l'utilisation des substances chimiques. Ilvise les substances en tant que telles, ainsi que celles présentes dans les préparations ou dans les articles. Les substances radioactives et les déchets ne sont pas visés par ce texte. L'Agence européenne des produits chimiques (ECHA), basée à Helsinki, a la charge des aspects techniques et administratifs liés au fonctionnement du dispositif REACH.
EDF est à la fois un fabricant de substances chimiques sur ses centrales nucléaires (fabrication de monochloramine et d'hypochlorite de sodium) et aussi un utilisateur de nombreuses substances chimiques sur tous ses sites d'exploitation. Dans ce cadre, en application du règlement $\mathrm{REACH}$, EDF démontre la conformité des scénarios d'exposition aux substances chimiques de ses travailleurs, et est engagé dans une démarche de substitution des substances les plus nocives pour l'homme et l'environnement.

\section{Règlement REACH}

Le règlement introduit des nouvelles procédures administratives que sont notamment :

- l'enregistrement : aucune substance soumise à enregistrement ne peut être fabriquée ni importée si elle n'a pas été enregistrée par le producteur ou I'utilisateur (" pas de données, pas de marché »). Ce sont les producteurs de substances et non plus les autorités publiques qui doivent prouver que les risques liés aux substances qu'ils produisent sont valablement maîtrisés : il s'agit alors d'un renversement de la charge de la preuve ;

- l'évaluation : l'évaluation permet à l'Agence européenne des produits chimiques de vérifier que l'industrie respecte ses obligations et évite les essais sur les animaux vertébrés inutiles ;

- I'autorisation : aucune substance soumise à la procédure d'autorisation (CMR 1 \& 2, PBT et $\left.v P \vee B^{*}\right)$ ne peut être utilisée si elle n'a pas fait l'objet d'une autorisation pour cet usage ;

- la restriction : c'est le filet de sécurité du système permettant de gérer les risques non couverts par ailleurs. Elle peut permettre l'interdiction pure et simple d'une substance sur le marché européen quel que soit son usage.

(*) CMR : cancérigène, mutagène, reprotoxique.

PBT : persistante, bioaccumulable, toxique.

$\mathrm{VPvB}$ : très persistante, très bioaccumulable.

\section{Réglementation française}

La réglementation française relative aux INB, dont font partie les centrales nucléaires d'EDF, repose sur des textes internationaux (conventions, protocoles...), des textes de droit communautaire (règlements, directives...) et sur des textes généraux de protection de l'environnement et de santé publique ou de lutte contre les nuisances (lois, décrets, arrêtés). Les autorisations délivrées pour chaque centrale nucléaire à EDF afin de prélever de l'eau et rejeter des effluents dans l'environnement découlent de ces nombreux textes dont les principaux sont présentés ci-après. Les textes propres à chaque centrale nucléaire d'EDF font l'objet du paragraphe 5.

En matière de lutte contre toutes les formes de pollutions (chimique, radioactive, thermique, microbiologique, etc.), deux approches réglementaires coexistent :

- la première vise la prévention des nuisances de toute nature par une approche dite «intégrée »: réglementation relative aux INB et aux ICPE ; 
- la seconde recherche la protection du milieu : réglementation relative à l'eau, à l'air...

Nota: La réglementation relative à l'information du public et les missions exercées par l'administration (notamment son rôle de police) sont respectivement abordées au chapitre 4 et au chapitre 6.

\subsection{RÉGLEMENTATION DANS LE DOMAINE NUCLÉAIRE}

\section{Code de l'environnement - partie législative : textes issus de la loi no 2006-686 du 13 juin 2006 relative à la transparence et à la sécurité en matière nucléaire (dite «TSN») codifiée}

La partie législative du titre IX du Livre $V$ découle principalement de la codification de la loi n²006686 du 13 juin 2006 relative à la transparence et à la sécurité en matière nucléaire (dite loi TSN), il s'agissait de la première loi spécifique dans le domaine du nucléaire en "raison des risques ou inconvénients qu'ils peuvent présenter pour la sécurité, la santé et la salubrité publiques ou la protection de la nature et de l'environnement »?

La loi «TSN » a été codifiée par l'ordonnance n 2012-6 du 5 janvier 2012 au sein du Code de l'environnement (livre ${ }^{\text {er }}$ et livre $\mathrm{V}$ ). Il convient donc de viser désormais les articles du Code de l'environnement et non plus ceux de la loi «TSN » (cf. annexe 5.3).

Les dispositions tirées de cette loi revêtent une grande importance car :

- elles identifient l'Autorité de sûreté nucléaire

$\left(\mathbf{A S N}^{2}\right)$ comme l'autorité de contrôle de prédilection du secteur. Il s'agit d'une autorité administrative indépendante ;

- elles donnent une base législative à la définition des INB et précise le régime juridique applicable aux autres installations implantées dans le périmètre d'une INB (cf. encart p. 81) ;

- elles fondent sa politique sur une approche intégrée de la réduction des nuisances et des pollutions ;

- elles reprennent les principes fondamentaux auxquels doivent satisfaire les activités présentant un risque d'exposition aux rayonnements ionisants (principes de justification, limitation, optimisation de la directive Euratom 96/29) :

- elles renforcent la transparence et notamment l'information du public ${ }^{3}$;
- elles assurent le fondement légal des commissions locales d'information ${ }^{4}$ et du Haut Comité pour la transparence et la sécurité nucléaire ${ }^{5}$; - elles renforcent les sanctions administratives ${ }^{6}$ et pénales ${ }^{7}$.

Avec la création de l'ASN, les compétences de sûreté nucléaire et de radioprotection au sein du gouvernement sont confiées à la Direction générale de la prévention et des risques (DGPR) placée sous l'autorité des ministres chargés de la Transition écologique (dont le portefeuille comprend actuellement celles du ministre chargé de la sûreté nucléaire) et de la Santé. Pour assister le ministre chargé de ces questions, la Mission de sûreté nucléaire et de radioprotection (MSNR) est intégrée à la Direction générale de la prévention des risques du ministère de la Transition écologique..

Pour mémoire, cette législation a introduit un changement notable en ce qui concerne la procédure de création et l'exploitation des INB. Jusqu'en 2006 (sous l'empire du décret de $1963^{\circ}$ ), deux types d'autorisation étaient nécessaires pour la création et pour les prélèvements d'eau et les rejets :

- une autorisation délivrée par le décret d'autorisation de création (DAC) portant sur les aspects de sûreté nucléaire ;

- une autorisation sous la forme d'un arrêté interministériel relatif aux prélèvements d'eau et aux rejets d'effluents (suivant le décret du 4 mai 1995).

Depuis 2006, l'autorisation principale est délivrée après l'organisation d'une enquête publique et obtention de l'avis de l'ASN : il s'agit du décret d'autorisation de création (DAC). Le DAC couvre notamment les aspects "sûreté », comme auparavant, mais aussi les prélèvements d'eau et les rejets (cf. \& 5). C'est pourquoi, depuis 2016, la création d'une INB nécessite désormais une évaluation environnementale ${ }^{10}$.

Des prescriptions complémentaires au DAC sont délivrées par l'ASN sur les prélèvements d'eau et les rejets, mais aussi sur les nuisances sonores et les moyens de contrôle de l'installation et de surveillance de ses effets sur l'environnement. Par ailleurs, le DAC d'une INB susceptible de rejeter des substances radioactives ne peut être accordé qu'après réception de l'avis de la Commission européenne au titre de l'article 37 du Traité EURATOM.

Dorénavant, la procédure administrative de création et de mise en service des INB est notamment prévue au chapitre III du titre IX du livre $V$ du Code de l'environnement.

1. Art. L. 593-1 du Code de l'environnement ex-art. 28-I de l'ex-loi.

2. Art. L. 592-1 à L. 592-40 du Code de l'environnement (ex art. 3 et 4 de l'ex-loi)

3. Art. L. 125-10-1 à L. 125-16 du Code de l'environnement (ex-art. 19 et 21 de l'ex-loi).

4. Art. L. 125-17-1 à L. 125-33 du Code de l'environnement (ex-art. 22 de l'ex-loi).

5. Art. L. 125-34 à L. 125-40 du Code de l'environnement (ex-art. 23 et 27de l'ex-loi)

6. Art. L. 596-3 à L. 596-23 du Code de l'environnement (ex-art. 41 et 44 de l'ex-loi)

7. Art. L. 596-10 à L. 596-12 du Code de l'environnement (ex-art. 53 et 56 de l'ex-loi)

8. Voir paragraphe suivant.

9. Voir paragraphe suivant.

10. Art. R. 122-2 du Code de l'environnement. 


\section{Principaux articles du Code de l'environnement relatifs aux INB, issus de la codification du " décret Procédures »}

Articles L. 593-7 à L. 593-10, R. 593-14 à R. 593-28, et R. 593-38 à R. 593-40 du Code de l'environnement : Dispositions relatives à la création d'une INB

Ces articles concernent :

- le dépôt du dossier de demande d'autorisation de création comportant un certain nombre de pièces (version préliminaire du rapport de sûreté, l'étude d'impact, le plan de démantèlement, etc.) ;

- les modalités d'instruction du dossier par I'ASN et le ministère en charge de la sûreté nucléaire ;

- l'organisation de l'enquête publique ;

- l'obtention du décret d'autorisation de création (définition du périmètre de l'INB, délai de mise en service de l'installation);

- les prescriptions à caractère technique de I'ASN pour l'application du DAC qui sont soumises pour avis au CoDERST et observations à la CLI (ex. prélèvements d'eau, rejet, surveillance de l'environnement, limitations des nuisances sonores, etc.).

\section{En particulier :}

L'article R. 593-17 indique les compléments attendus de l'étude d'impact d'une INB au regard du contenu de droit commun (R. 122-5 CE) (cf. chapitre 8).

L'article R. 593-38 précise la procédure en vue d'établir et modifier les prescriptions (sous la forme de décision de I'ASN). Dans le cas où les prescriptions concernent les limites de rejet, celles-ci doivent être homologuées par le ministre en charge de la Sûreté.

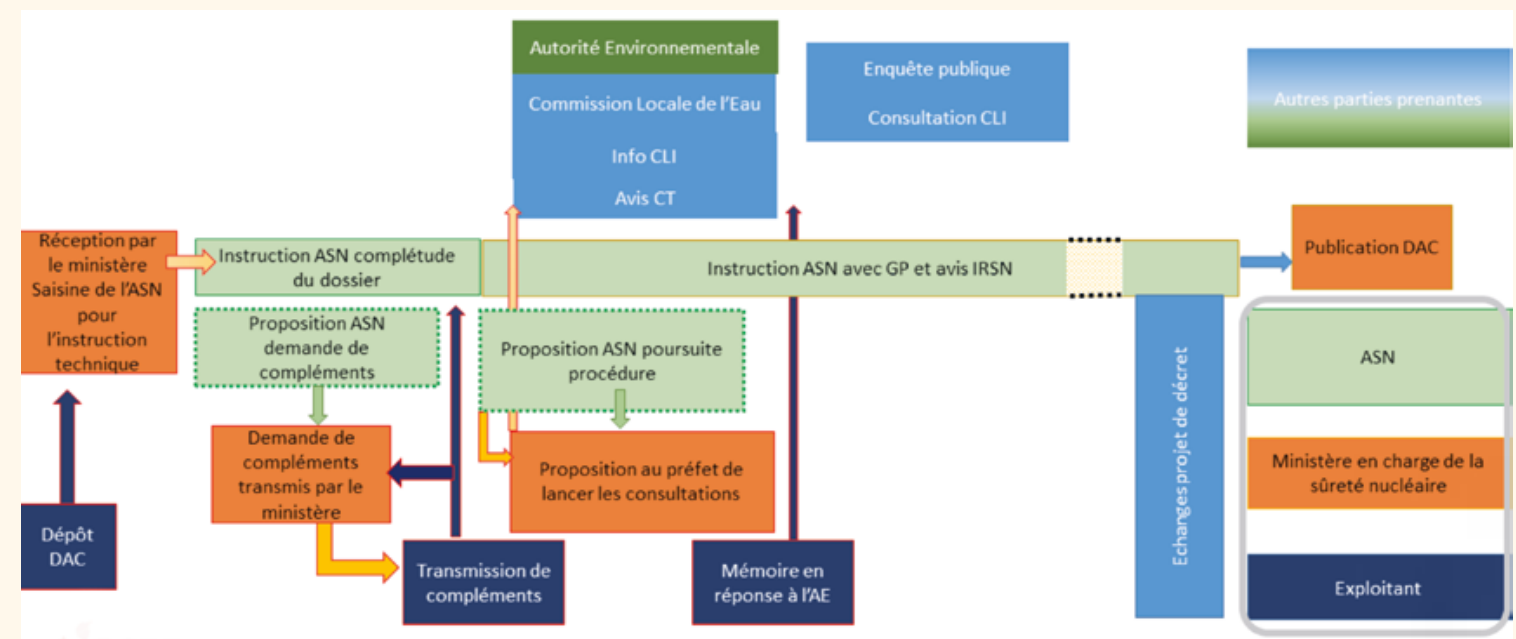

Articles L. 593-11 et R. 593-29 à R. 593-37 du Code de l'environnement : Dispositions relatives à la mise en service d'une INB

Ces articles portent notamment sur :

- le dépôt d'un dossier comprenant notamment le rapport de sûreté (mise à jour de la version préliminaire), les règles générales d'exploitation (RGE), le plan d'urgence interne en cas d'incident ou d'accident (PUI), la mise à jour de l'étude d'impact le cas échéant ;

- la délivrance de l'autorisation par une décision de I'ASN qui fait l'objet d'une mention au Bulletin officiel de l'ASN. Elle est notifiée à l'exploitant et communiquée au ministre chargé de la Sûreté nucléaire et au préfet.

Articles L. 593-15 et R. 593-55 à R. 593-61 du Code de l'environnement : Dispositions relatives aux modifications d'exploitation

Sont concernées, les modifications ayant une incidence sur les intérêts protégés, de ses modalités d'exploitation autorisées, des éléments ayant conduit à l'autorisation de création ou de mise en service. Ces modifications sont soumises soit à déclaration auprès de l'ASN (articles R. 593-59 à R. 593-61), soit autorisation (articles R. 593-55 à R. 593-58), selon leur importance, et peuvent être soumises à consultation du public. La décision n²017-DC-0616 de l'ASN du 30 novembre 2017 relative aux modifications notables des INB fixe la liste des modifications notables soumises à autorisation et déclaration auprès de l'ASN. 
Articles L. 593-14 et R. 593-41 à R. 593-54 du Code de l'environnement : Disposition relatives à la modification du DAC initial

Ces articles définissent les démarchent à suivre lors du changement d'exploitant, la création d'une installation nucléaire de base par séparation ou par réunion d'installations existantes, de modifications substantielles, etc.

Concernant les modifications substantielles (L. 593-14), ces dernières font l'objet d'une procédure similaire à celle d'autorisation de création, selon la procédure prévue par les articles L. 593-7 à L. 593-12. L'article R. 593-47 énonce les cas considérés comme des modifications substantielles (changement de nature, modification des éléments essentiels de l'autorisation de création, ajout d'une nouvelle INB dans le périmètre, etc.).

Articles L. 593-3, L. 593-33 et R. 593-86 à R. 593-88 du Code de l'environnement : Dispositions relatives aux autres installations situées à l'intérieur et à l'extérieur du périmètre de l'INB

Ces articles précisent l'articulation des régimes INB et ICPE

Au sein du périmètre de I'INB :

- les équipements et installations nécessaires à l'exploitation de l'INB sont réputés faire partie de cette installation, et soumis au régime et à la réglementation applicable aux INB ;

- les équipements et installations sans lien (non nécessaires) avec I'INB relevant des nomenclatures ICPE et IOTA restent soumis à leur régime avec une compétence de l'ASN pour prendre les mesures individuelles relatives à ces équipements et installations.

En dehors du périmètre de l'INB, les matériels, équipements et activités sont réglementés par les textes généraux relatifs aux ICPE et IOTA, à la protection de l'environnement et à la santé publique.

Code de l'environnement - partie réglementaire : issue notamment du décret $n^{\circ}$ 2007-1557 modifié du 2 novembre 2007 dit « décret Procédures», articles R. 593-1 et suivants.

Le « décret Procédures », codifié au sein du Code de l'environnement, portait principalement sur les procédures administratives à suivre pour la création d'une INB, sa mise en service, son fonctionnement, sa modification, sa mise à l'arrêt définitif, et son démantèlement. Les dispositions relatives aux ICPE et IOTA² dans le périmètre de l'INB mais non nécessaires à son exploitation sont partiellement régies par les dispositions du titre IX (l'ASN étant l'autorité administrative compétente) mais également par les dispositions communes à ces installations, ainsi que le souligne l'article L. 593-33 du code précité.

L'arrêté du 7 février 2012 fixant les règles générales relatives aux installations nucléaires de base (arrêté «INB ») modifié

L'arrêté du 7 février 2012 modifié établit les règles générales applicables aux INB. Cet arrêté couvre un champ très large puisqu'il vise à protéger les « intérêts » mentionnés à l'article L. 593-1 du Code de l'environnement. Il concerne le management de la sûreté, la maîtrise des risques d'accident, la maîtrise des nuisances et de l'impact sur la santé et sur l'environnement, la gestion des déchets, les situations d'urgence.

Il s'applique à toutes les phases de la « vie » d'une INB de la conception jusqu'au démantèlement, en passant par la construction, le fonctionnement, l'entretien et la surveillance des installations et la mise à l'arrêt définitif.

L'arrêté reprend et actualise certaines dispositions de l'arrêté du 10 août 1984 relatif à la qualité de la conception, de la construction et de l'exploitation des INB (dit " arrêté qualité »), l'arrêté du 26 novembre 1999 fixant les prescriptions techniques générales relatives aux limites et modalités des prélèvements et des rejets soumis à autorisation, effectués par les INB et l'arrêté du 31 décembre 1999 fixant la réglementation technique générale destinée à prévenir et limiter les nuisances et les risques externes résultant de l'exploitation des INB (cf. encart). Ces textes ont été abrogés

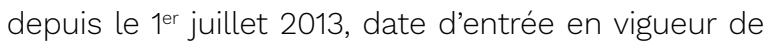
l'arrêté du 7 février 2012 (sauf dispositions particulières).

Il transpose aux INB des dispositions applicables aux ICPE/IOTA (cf. arrêtés listés en annexe II de l'arrêté INB), notamment en matière de limites de rejet et de surveillance de l'environnement et fournit, par ailleurs, des définitions sur des termes-clés tels que «fonctionnement normal » ou « en mode dégradé », " effluent », « événements significatifs », « incident ou accident », « élément important pour la protection » (EIP), « activité importante pour la protection » (AIP)...

Ces exigences sont complétées par des décisions réglementaires à caractère technique de l'ASN. 
CODE DE L'ENVIRONNEMENT ET DÉCRETS D’APPLICATION

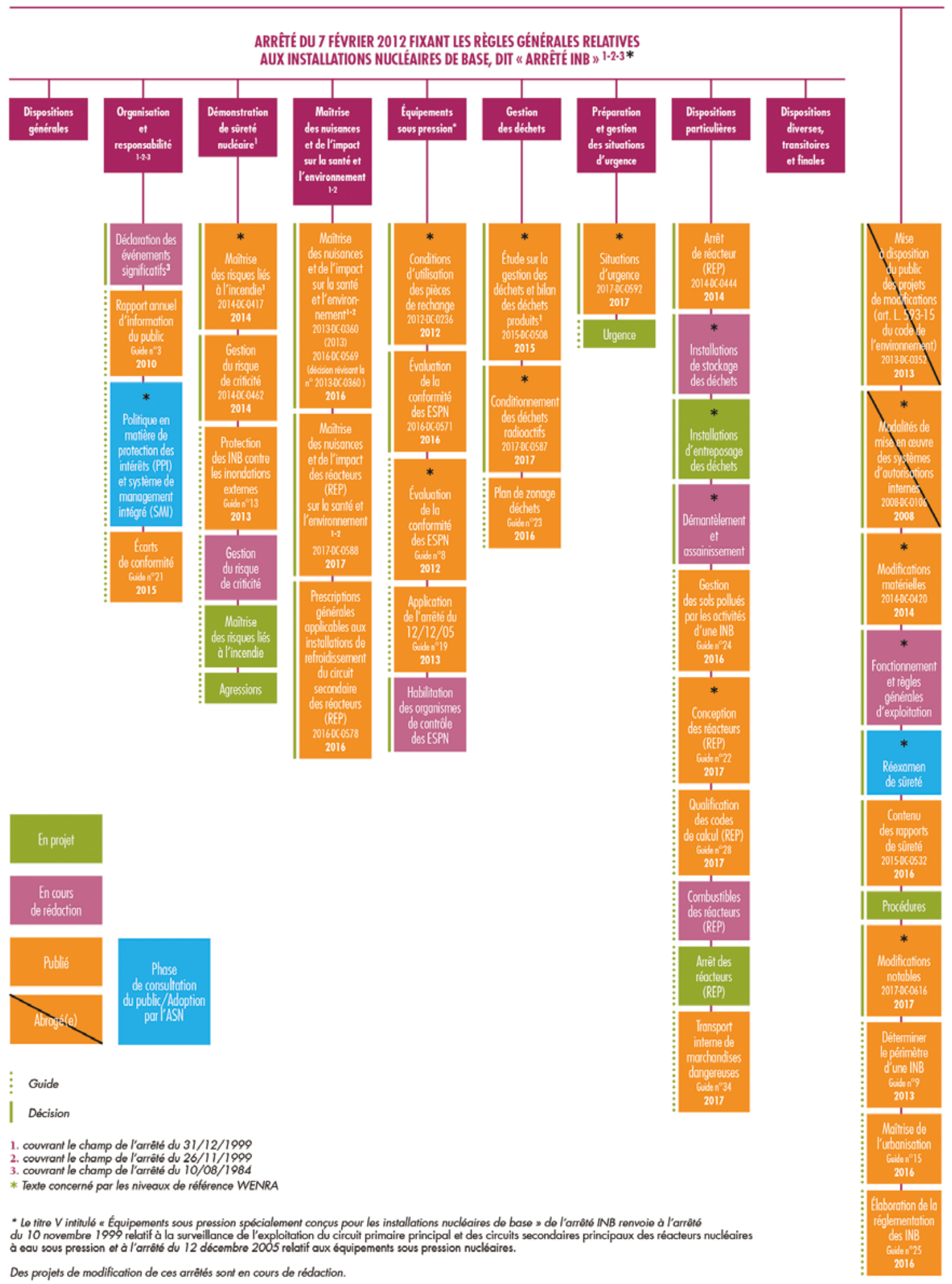

Logigramme des décisions de l'ASN et leur articulation avec l'arrêté INB Source : site Internet de l'ASN 


\section{Arrêté 7 février 2012 dit «INB » fixant les règles générales relatives aux installations nucléaires de base modifié}

En établissant un lien entre sûreté nucléaire, radioprotection du public et protection de l'environnement, cet arrêté constitue, avec la réglementation relative à l'eau (IOTA) et aux ICPE, la base réglementaire sur laquelle les dispositions propres à chaque centrale nucléaire sont établies s'agissant des prélèvements d'eau et des rejets (cf. § 5). L'arrêté est structuré en neuf titres, dont notamment :

Le titre II Organisation et la responsabilité concernant :

- la politique en matière de protection des intérêts mentionnés par la loi (sécurité, santé et salubrité publiques, protection de la nature et de l'environnement) que l'exploitant tient à la disposition de l'ASN dans un document ;

- le système de gestion intégrée que l'exploitant met en place afin de respecter les exigences précitées. Ce système précise par ailleurs les dispositions mises en œuvre en termes d'organisation et de ressources de tout ordre pour répondre aux objectifs de protection des intérêts mentionnés par la loi. Il comporte également des dispositions relatives aux traitements des éventuels écarts, au retour d'expérience et à la définition d'indicateurs d'efficacité et de performance ;

- I'amélioration continue des pratiques;

- les modalités d'information du public.

Le titre IV Maîtrise des nuisances et de l'impact sur la santé et l'environnement avec des dispositions sur :

- les prélèvements d'eau et des rejets d'effluents dans l'air et dans l'eau ;

- la surveillance ;

- la prévention des nuisances (odeur, bruit...) ;

- I'information de l'autorité de contrôle.

Le titre IX Dispositions transitoires et finales

\subsection{RÉGLEMENTATION DES INSTALLATIONS CLASSÉES POUR LA PROTECTION DE L'ENVIRONNEMENT (“ ICPE »)}

\author{
Code de l'environnement : textes issus de la loi \\ $n^{\circ} 76-663$ du 19 juillet 1976 relative aux ICPE \\ (prévention et nuisances), articles L. 181-1 et suivants, \\ L. 511-1 et suivants, et R. 511-9 et suivants,
}

Les centrales nucléaires sont concernées par la réglementation applicable aux ICPE pour tous les équipements et installations non nécessaires à leur exploitation, ainsi que pour les équipements et installations situés à l'extérieur du périmètre de l'INB (cf. §. 5).

La réglementation sur les ICPE est fondée sur une approche intégrée de la protection de l'environnement, elle s'intéresse aussi bien aux problèmes de pollution de l'eau et de l'air qu'aux risques, aux déchets et aux nuisances diverses (odeur, bruit...) résultant des activités industrielles ou autres inscrites sur une liste (nomenclature) fixée par annexe à l'article R. 511-9 du Code de l'environnement.

La nomenclature ICPE soumet les installations classées à autorisation, enregistrement ou déclaration suivant la gravité des dangers ou inconvénients que peut présenter leur exploitation. Ce régime a été modifié par la réforme de l'autorisation environnementale (ordonnance du 26 janvier 2017) qui fusionne dans une même décision administrative, les procédures d'instruction et de délivrance de différentes autorisations prévues au titre du Code de l'environnement.

\subsection{TEXTES SUR L'EAU ET LES MILIEUX AQUATIQUES}

Les textes législatifs et réglementaires relatifs à l'eau sont importants, s'agissant des prélèvements d'eau et des rejets d'effluents des centrales nucléaires, car les textes (arrêté d'autorisation, décisions ASN) fixant les prescriptions et les limites applicables aux centrales nucléaires s’y réfèrent (cf. §. 5).

\section{Loi n 2006-1772 du 30 décembre 2006 sur l'eau et} les milieux aquatiques, dite "LEMA », codifiée aux articles L. 210-1 et suivants et R. 211-1 et suivants du Code de l'environnement.

Cette loi et les textes qui en découlent ont rénové le cadre global défini par les lois antérieures de 1964 et de 1992. Ceux-ci ont pour principaux objectifs de :

- répondre aux enjeux de la politique européenne de l'eau poursuivant l'objectif de " bon état » des eaux défini par la directive européenne (DCE) en fixant notamment des objectifs de qualité d'eau pour chaque bassin hydrographique dans les schémas directeurs d'aménagement de gestion de l'eau (SDAGE) ;

- prendre en compte l'enjeu sociétal en proclamant un droit à l'eau pour tous ;

- répondre aux attentes du public et des usagers de l'eau en matière d'information ;

- préserver les milieux aquatiques par une gestion en quantité et qualitative de l'eau, ce qui suppose d'assurer les continuités écologiques tant pour 
les migrations de certaines espèces que pour le transit des sédiments (cf. encart sur les objectifs de débit).

Volet organisationnel (cf. chapitre 6)

- Principe de la gestion par bassin hydrographique' ${ }^{1}$ et l'idée de gouvernance à laquelle sont associés les usagers.

- Composition des comités de bassin : 40 \% pour les collectivités territoriales, $40 \%$ pour les usagers (pour moitié les usagers économiques comme les entreprises, et pour moitié les usagers non économiques), $20 \%$ pour l'État.

- Identification d'un Organe institutionnel pour le secteur : cet organe historique était l'Office national de l'eau et des milieux aquatiques (ONEMA) incorporé dans l'Agence française pour la biodiversité par la loi Biodiversité du 8 août 2016 qui reprenait ses missions ${ }^{2}$, puis par l'Office français de la biodiversité créé par la loi n²019-773 du 24 juillet 2019.

- Identification des missions des six agences de l'eau qui concernent en particulier la mise en œuvre des SDAGE et de leur déclinaison au niveau local dans les SAGE élaborés par les commissions locales de l'eau (CLE).
- Portée juridique des schémas d'aménagement et de gestion de l'eau (SAGE) renforcée : ceux-ci sont opposables aux tiers.

Volet financier

- Le Parlement dispose du pouvoir de fixer les règles concernant, l'assiette, les taux plafond, les modalités de recouvrement ainsi que les critères qui permettront aux comités de bassin de moduler les taux des redevances notamment celles portant sur les prélèvements d'eau et les rejets d'effluents (cf. chapitre 6).

\section{Nomenclature « eau»}

À l'image de la nomenclature des ICPE, il existe une nomenclature " eau » qui établit la liste des installations, ouvrages, travaux et aménagements (IOTA) soumis à autorisation ou déclaration (R. 214-1 du Code de l'environnement). Comme pour les ICPE, certains projets de IOTA peuvent être soumis à évaluation environnementale ou soumis à examen au cas par cas.

\section{Objectifs de débits d'étiage, d'alerte et de crise définis dans les SDAGE}

La disponibilité des ressources en eau étant variable dans le temps et suivant les lieux, les objectifs de débit des SDAGE sont établis en distinguant :

- les conditions pour lesquelles il est possible et nécessaire de concilier normalement les besoins des usagers et les exigences des milieux ;

- de celles, plus exceptionnelles, à partir desquelles des défaillances apparaissent et où il faut gérer les risques de pénurie et chercher à les réduire en proposant des mesures structurantes.

Dans les SDAGE, il existe un réseau de points stratégiques, appelés points nodaux, où sont fixées, outre le débit d'objectif d'étiage (DOE), les valeurs des débits seuils d'alerte (DSA) et de crise (DSR). Les points nodaux correspondent à des stations de mesure de débit situées, soit à l'aval des unités hydrographiques, soit en d'autres points intéressants pour leur caractère singulier (principale confluence, prise d'eau, rejet, barrage...).

Équilibre « besoins - ressources en eau »

Le débit objectif d'étiage (DOE) est le débit moyen mensuel permettant de satisfaire tous les besoins en moyenne huit années sur dix et d'atteindre le bon état des eaux. Sa valeur est fixée au débit moyen mensuel de récurrence 5 ans (QMNA 5), qui correspond à la plus faible moyenne sur l'année des débits mensuels d'étiage de fréquence quinquennale et qui constitue la référence pour les objectifs de qualité et pour l'application de la réglementation en matière de rejet et de prélèvement.

\section{Gestion de crise en période de sécheresse}

Le débit seuil d'alerte (DSA) est le débit moyen journalier en dessous duquel un usage ou une fonction de la rivière ne peut plus être assuré dans des conditions normales. Ce seuil constitue un signal à partir duquel des dispositions à caractère volontaire ou faiblement contraignant peuvent être envisagées de manière à ne pas atteindre le niveau de crise (DCR), si la situation s'aggrave.

Le débit de crise (DCR) est le débit moyen journalier en dessous duquel ne sont plus garantis les besoins indispensables en eau potable pour la vie humaine et animale, ceux relatifs à la survie des espèces piscicoles les plus intéressantes et à l'activité économique. 


\section{Évolutions de la réglementation dans le domaine de l'eau}

1964: Loi n 64-1245 du 16 décembre 1964 relative au régime et à la répartition des eaux et à la lutte contre leur pollution. 1992 : Loi n` 92-3 du 3 janvier 1992 sur l'eau a profondément modifié la loi de 1964 dont elle conserve quelques articles relatifs à la création du Comité national de l'eau, aux agences financières de bassin devenues agences de l'eau, et aux redevances. Elle a notamment pour objectifs une gestion globale et équilibrée de l'eau, la lutte contre les pollutions et le gaspillage et instaure pour cela une police administrative unique de contrôle de la qualité des eaux et du niveau de la ressource en eau et met en place deux systèmes de planification des usages de l'eau, à savoir, les SDAGE à l'échelle du bassin, les SAGE au niveau du cours d'eau.

2000 : Directive 2000/60/CE du 23 octobre 2000 établissant un cadre pour une politique communautaire dans le domaine de l'eau (« DCE »).

2003-2004 : Débat national et décentralisé sur l'eau avec toutes les parties prenantes.

2004 : Transposition en droit français de la DCE, par la loi n²004-338 du 21 avril 2004.

2006 : Loi n`2006-1772 du 30 décembre 2006 sur l'eau et les milieux aquatiques («LEMA ») qui rénove le cadre global défini par les lois de 1964 et 1992.

Quelques textes découlant de l'application de la «LEMA »:

- décret n²007-833 du 11 mai 2007 relatif au Comité national de l'eau ;

- décret n² 2007-443 du 25 mars 2007 relatif à l'Office national de l'eau et des milieux aquatiques ;

- décret n²007-980 du 15 mai 2007 relatif aux comités de bassin ;

- arrêté du 25 janvier 2010 relatif aux méthodes et critères d'évaluation de l'état écologique, de l'état chimique et du potentiel écologique des eaux de surface en application de l'art. R. 212-10, R. 212-11 et R. 212-18 du Code de l'environnement (modifié par l'arrêté du 27 juillet 2015) ;

- arrêté du 25 janvier 2010 établissant le programme de surveillance de l'état des eaux en application de l'art. R. 212-22 du Code de l'environnement (modifié en 2011, 2015 et 2018) ;

- décret n²015-450 du 20 avril 2015 relatif au Comité national de l'eau ;

- décret n²018-847 du 4 octobre 2018 relatif aux schémas directeurs d'aménagement et de gestion des eaux et schémas d'aménagement et de gestion des eaux;

- loi Biodiversité du 8 août 2016 et loi n 2019-773 du 24 juillet 2019 portant création de l'Office français de la biodiversité, modifiant les missions des fédérations des chasseurs et renforçant la police de l'environnement.

\subsection{TEXTES SUR L'AIR}

La réglementation française reprend les exigences de la réglementation européenne sur les substances appauvrissant la couche d'ozone (règlement 2000/2037/CE du 29 juin 2000 qui interdit l'utilisation des CFC depuis 2001 et celle des HCFC à partir de 2015, pour la maintenance et l'entretien des équipements de réfrigération et de climatisation, le règlement n’ 1005/2009 du 16/09/09 relatif à des substances qui appauvrissent la couche d'ozone a abrogé ce règlement et prend sa place) et les gaz à effet de serre (règlement 2006/842/CE du 17 mai 2006, abrogé par le règlement (UE) n० 517/2014 - dit F-Gas - du 16/04/14 relatif aux gaz à effet de serre fluorés et abrogeant le règlement (CE) n 842/2006) utilisés dans les systèmes de climatisation et de réfrigération. Les INB et les centrales nucléaires sont tenues de s'y conformer.

Les centrales nucléaires sont également concernées par la loi n 96-1236 du 30 décembre 1996 sur l'air et l'utilisation rationnelle de l'énergie dite " LAURE », aujourd'hui codifiée par l'ordonnance n²000-914 du 18 septembre 2000 au sein du Code de l'environnement (titre II Air et atmosphère), qui demande d'étudier l'impact des activités humaines sur la santé publique. Ceci vise les impacts des rejets industriels et en particulier, pour ce qui est des centrales nucléaires, les émissions atmosphériques gazeuses issues d'opérations de lessivage chimique (ammoniac) ou les gaz d'échappement des turbines à combustion (TAC) de secours et les moteurs diesels de secours définis, par ailleurs, dans la rubrique ICPE n 2910.

La France a également ratifié l'amendement au protocole de Montréal adopté à Kigali le 15 octobre 2016 relatif à des substances appauvrissant la couche d'ozone,

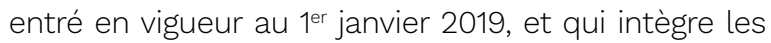
hydrofluorocarbures dans le champ de ce protocole?.

Enfin, la directive (UE) 2015/2193 du Parlement européen et du Conseil du 25 novembre 2015 relative à la limitation des émissions de certains polluants dans l'atmosphère en provenance des installations de combustion moyennes, qui fixe des valeurs limites d'émission pour les installations de combustion de puissance comprise entre 1 et $50 \mathrm{MW}$, a été transposée en droit français par le décret n²018-704 du 3 août 2018 modifiant la nomenclature des installations classées et certaines dispositions du Code de l'environnement et 5 arrêtés ministériels de prescriptions générales, entrés en vigueur le 20 décembre 2018.

1. Le décret no 2019-708 du 4 juillet 2019 portant publication de l'amendement au protocole de Montréal du 16 septembre 1987 relatif à des substances qui appauvrissent la couche d'ozone, adopté à Kigali le 15 octobre 2016, a intégré cet amendement au droit français. 


\section{Textes propres à chaque centrale nucléaire d'EDF}

Les textes réglementaires du régime INB (décret, arrêté, décision) évoluent continuellement en devenant, au fur et à mesure, plus complexes et plus contraignants pour l'exploitant d'INB.

Pour s'assurer du respect de la réglementation dans sa déclinaison opérationnelle, l'exploitant met en place une organisation assurant un suivi des évolutions réglementaires, dénommé « contrôle de conformité réglementaire », afin d'adapter en permanence ses pratiques aux nouvelles exigences. L'exploitant décline les exigences réglementaires environnement dans un outil informatique dédié.

\subsection{ARTICULATION DES PRINCIPAUX TEXTES CONDUISANT AUX AUTORISATIONS DE PRÉLÈVEMENTS D’EAU ET DE REJETS (cf. figure 2)}

Sur la base de la réglementation en vigueur, l'administration délivre des autorisations et l'ASN fixe par décision des prescriptions réglementant notamment:

- les prélèvements et la consommation d'eau ;

- les rejets dans l'environnement des effluents liquides et gazeux ;

- les installations relevant d'un régime autonome (ICPE, IOTA) mais se trouvant dans le périmètre de l'INB et étant nécessaires à cette installation.
Ces autorisations sont délivrées, sous la forme de décret accordé par le ministre ou de décisions de l'ASN, après instruction de la demande d'autorisation déposée par le pétitionnaire.

Depuis 2006, le DAC couvre les aspects sûreté mais aussi, notamment, protection du public et de l'environnement. Ce décret est complété par deux décisions de l'ASN, l'une fixant les limites de rejets d'effluents dans l'environnement, l'autre portant sur les modalités de prélèvements et de consommation d'eau et de rejets d'effluents. La décision de l'ASN fixant les limites de rejet est homologuée par un arrêté signé du ministre chargé de la sûreté nucléaire. À cela s'ajoutent notamment les règles générales d'exploitation (RGE) rédigées par l'exploitant et validées par l'ASN avant la mise en service des installations et, le cas échéant, à chaque modification de celles-ci.

Par ailleurs, une centrale dispose d'autorisations délivrées par l'ASN ou le préfet pour les installations relevant des réglementations «ICPE », « eau », « air »... ou par l'administration chargée du domaine fluvial ou maritime pour l'occupation du domaine public. En outre, les autorisations délivrées pour les centrales frontalières doivent tenir compte des conventions ou accords internationaux passés entre les pays concernés.

Fig. 2 Articulation des principaux textes.

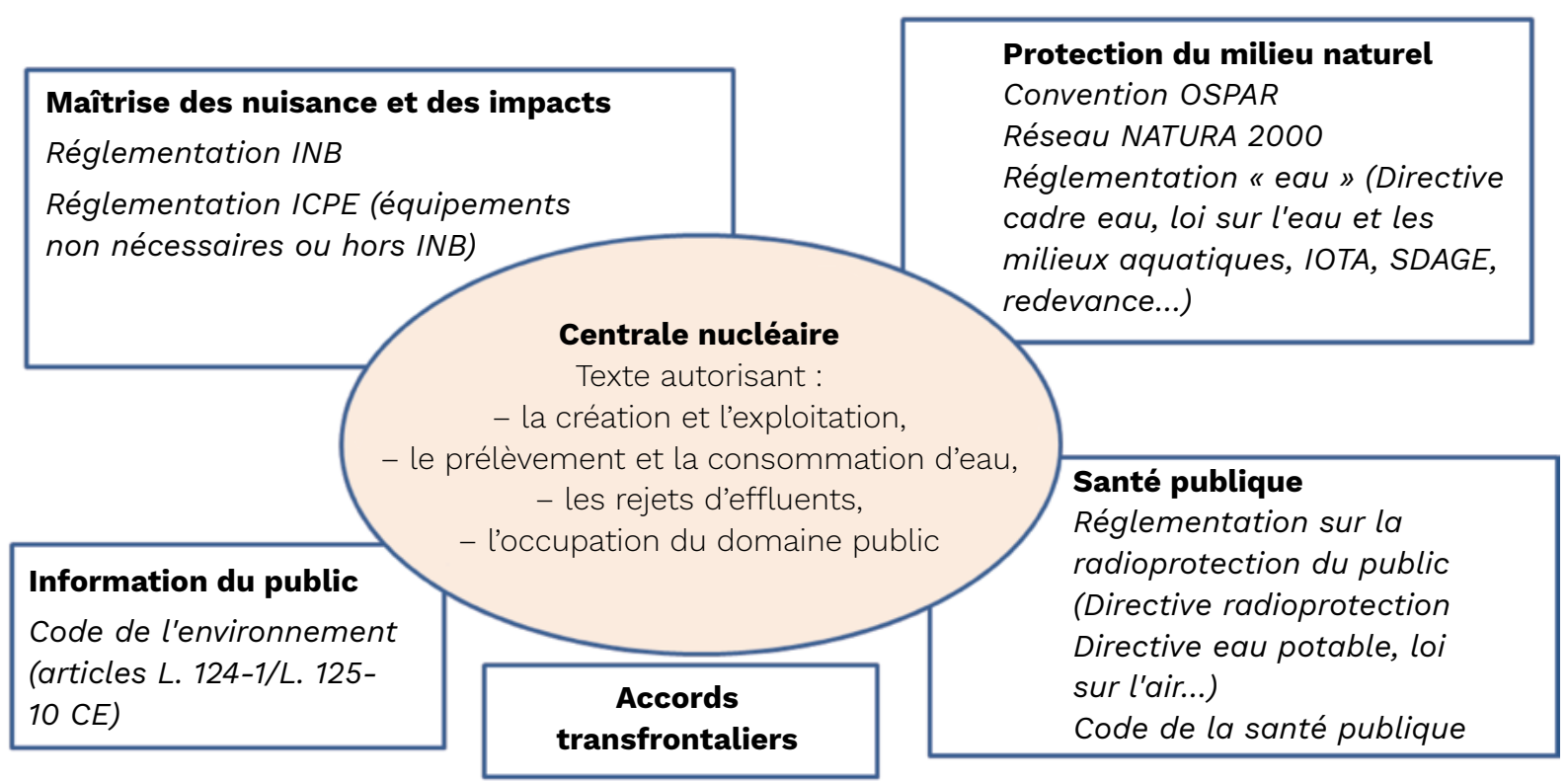




\subsection{TEXTES PROPRES À CHAQUE CENTRALE NUCLÉAIRE D'EDF}

\section{Prélèvement d'eau et rejets d'effluents}

La procédure administrative à engager par l'exploitant afin de solliciter les autorisations nécessaires est définie par la réglementation (Code de l'environnement). À l'issue de l'instruction de cette procédure, l'administration compétente délivre les autorisations au moyen des textes ci-après (cf. figure 3) :

- le décret d'autorisation de création (DAC) : ce décret précise, pour ce qui est de la maîtrise des impacts sur le public et l'environnement, que « Toute disposition est prise dans la conception et l'exploitation de l'installation, en particulier par l'utilisation des meilleures technologies industrielles disponibles à un coût économiquement acceptable, pour limiter les prélèvements d'eau douce et l'impact des rejets sur les populations et l'environnement. L'exploitant assure la réalisation périodique de contrôles de l'environnement » (ex. décret d'autorisation de création de Flamanville 3 du 10 avril 2007);

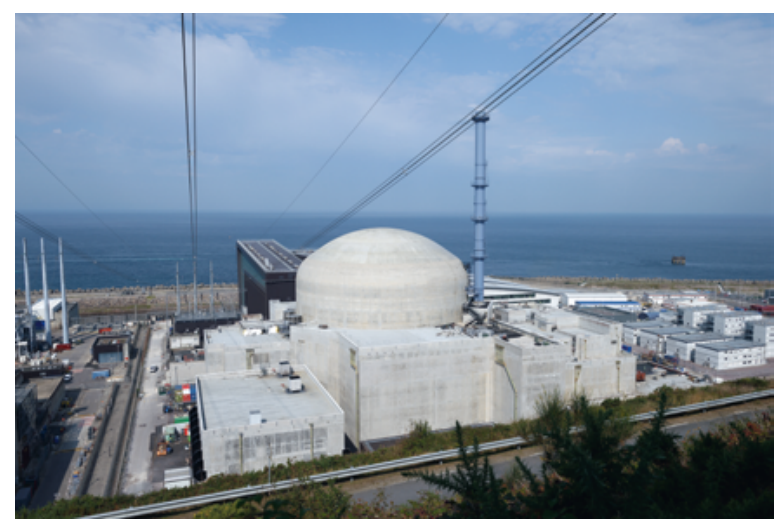

Le chantier EPR Flamanville 3, 27 août 2019 (c) EDF - Antoine Soubigou

\footnotetext{
- la décision de l'ASN fixant les limites de rejet dans l'environnement des effluents liquides et gazeux : Ces limites concernent:

- les rejets des effluents radioactifs gazeux,

- les rejets d'effluents radioactifs liquides,

- les rejets chimiques liquides et gazeux,

- les rejets thermiques.
}

Cette décision est homologuée par un arrêté signé du ministre chargé de la sûreté nucléaire ;
- la décision de l'ASN fixant les prescriptions relatives aux modalités de prélèvements et de consommation d'eau et de rejets dans l'environnement des effluents liquides et gazeux porte notamment sur :

- les prélèvements et la consommation d'eau,

- les rejets d'effluents dans l'environnement,

- la surveillance de l'environnement autour du site,

- le contrôle exercé par les autorités,

- l'information du public.

\section{Prélèvement d'eau et rejets d'effluents}

Les autorisations de prélèvement d'eau et de rejets accordées aux centrales nucléaires sont délivrées par l'ASN et relèvent des dispositions de l'article R. 593-38 du Code de l'environnement, qui prescrit les conditions dans lesquelles l'installation peut procéder à des prélèvements d'eau ou à des rejets directs ou indirects d'effluents dans le milieu ambiant.

\section{- l'autorisation de mise en service et le dossier associé :}

L'article R. 593-30 du Code de l'environnement prévoit que l'exploitant adresse à l'ASN un dossier contenant «les règles générales d'exploitation que l'exploitant prévoit de mettre en œuvre, dès la mise en service de l'installation, pour la protection des intérêts mentionnés à l'article L. 593-1 ». Auparavant orientées principalement "sûreté ». S'agissant des RGE, elles couvrent dorénavant un champ élargi, puisqu'elles portent également sur la sécurité, la santé et la salubrité publique ou la protection de la nature et de l'environnement (intérêts protégés visés à l'article L. 593-1 du Code de l'environnement).

Elles constituent, avec la mise à jour de la version préliminaire du rapport de sûreté et les autres documents listés à l'article R. 593-30 du Code de l'environnement pré-cité (mise à jour ou actualisation le cas échéant de l'étude d'impact notamment), l'engagement que prend l'exploitant vis-à-vis de l'ASN pour exploiter ses installations dans de bonnes conditions. Le chapitre relatif à l'environnement couvre un champ plus large que les seuls prélèvements d'eau et rejets d'effluents puisqu'il porte aussi sur la gestion des déchets et la maîtrise des nuisances (bruit, odeurs...) et les risques d'incendie et de foudre.

Fig. 3 - Les autorisations du régime INB.

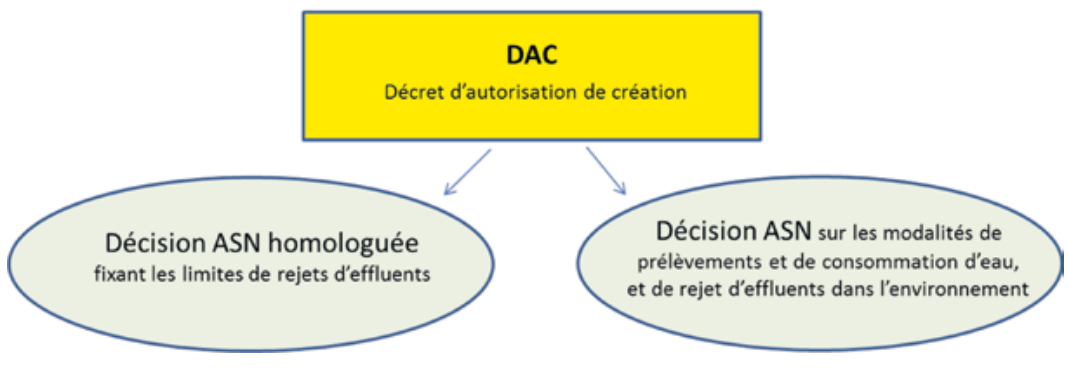




\section{Évolution des autorisations en matière de prélèvements d'eau et de rejets}

Autorisations délivrées après le décret $n^{\circ}$ 2019-190 du 14 mars 2019 codifiant les dispositions applicables aux installations nucléaires de base issues de la loi «TSN » de 2006 et de son décret d'application du 2 novembre 2007

- Décret d'autorisation de création (DAC)

- Décision de l'ASN, homologuée par arrêté du ministre chargé de la sûreté nucléaire, fixant les limites de rejets dans l'environnement des rejets liquides et gazeux,

- Décision de l'ASN fixant les prescriptions relatives aux modalités de prélèvements et de consommation d'eau et de rejets dans l'environnement d'effluents liquides et gazeux.

Autorisations délivrées avant 2006 au titre du décret du 4 mai 1995 (concerne plusieurs centrales) 1995 à 2006 au titre du décret nº 95-540 du 4 mai 1995 modifié relatif aux INB

- Décret d'autorisation de création (DAC) comprenant de prescriptions générales sur les rejets,

- Arrêté interministériel unique réglementant les prélèvements d'eau et les rejets.

\section{Autres prescriptions applicables}

Pour certaines activités, les centrales nucléaires sont réglementées au moyen :

- d'arrêtés préfectoraux pour les activités réalisées à l'extérieur du périmètre de l'INB (ex. opération de dragage et curage du cours d'eau qui relève du Code de l'environnement, réglementation ICPE/IOTA) ;

- de prescriptions particulières de l'ASN sous forme de décision en ce qui concerne les installations et des équipements situés à l'intérieur de l'INB mais non nécessaires à son fonctionnement (réglementation ICPE/IOTA).

\section{Occupation du domaine public fluvial et maritime}

Pour les centrales nucléaires dont les ouvrages de prises d'eau et de rejets sont construits sur le domaine public, il est nécessaire d'obtenir de la part de l'administration compétente une autorisation ou une concession d'occupation du domaine public au titre du Code général de la propriété des personnes publiques (cf. tableau I).

Tableau I Administration chargée du domaine public (cf. chapitre 6).

\begin{tabular}{|c|c|c|c|}
\hline & $\begin{array}{c}\text { Cours d'eau navigables } \\
\text { (centrales sur le Rhône, la Moselle, } \\
\text { la Meuse, la Seine, la Garonne) }\end{array}$ & $\begin{array}{c}\text { Cours d'eau non navigable } \\
\text { (centrales du Val de Loire) }\end{array}$ & $\begin{array}{c}\text { Domaine maritime } \\
\text { Centrales marines ou en estuaire }\end{array}$ \\
\hline $\begin{array}{c}\text { Administration } \\
\text { compétente }\end{array}$ & Voies navigables de France (VNF) & $\begin{array}{c}\text { Direction départementale } \\
\text { des territoires (DDT) }\end{array}$ & $\begin{array}{c}\text { Port autonome ou } \\
\text { Direction départementale } \\
\text { des territoires et de la mer (DDTM) }\end{array}$ \\
\hline
\end{tabular}

\footnotetext{
POUR ALLER PLUS LOIN

- http://www.legifrance.gouv.fr

- https://www.ecologique-solidaire.gouv.fr/

- http://www.asn.fr

- http://www.eau-seine-normandie.fr/

- https://aida.ineris.fr/
} 


\section{Annexe 5.1}

\section{Tableau récapitulatif des principaux textes réglementaires}

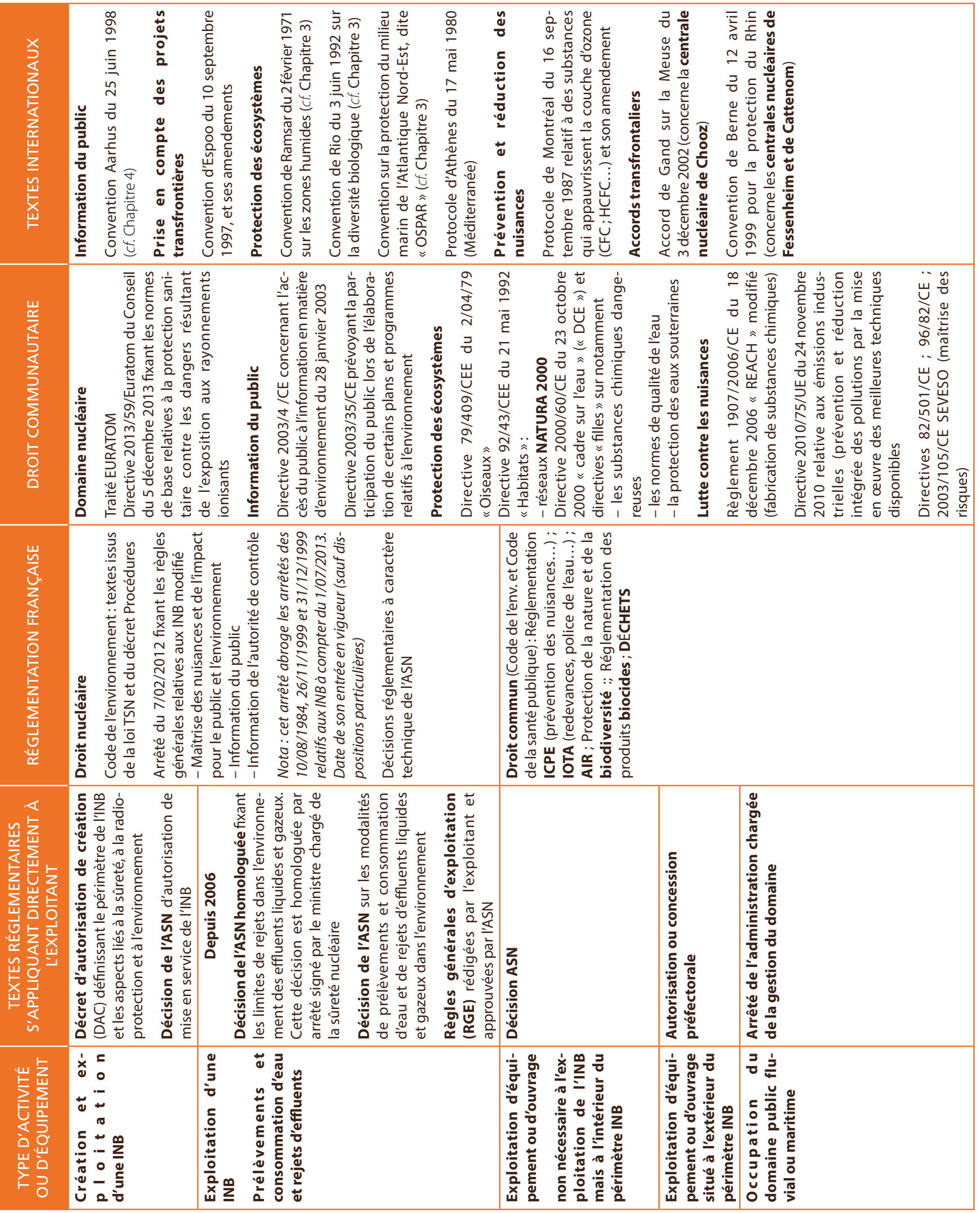




\section{Annexe 5.2}

\section{Nature des différents types de textes législatifs et réglementaires}

DROIT INTERNATIONAL DE L'ENVIRONNEMENT

Traité : Acte écrit par lequel des États établissent des règles créant des droits et obligations. Il doit être ratifié (par le Parlement ou par référendum en France) pour produire ses effets de droit et devenir ainsi un instrument obligatoire liant les parties signataires.

Convention : Accord de volonté entre deux ou plusieurs États sur un objet ou fait précis et qui oblige les signataires au respect de son contenu (ex. Convention sur la protection du milieu marin de l'Atlantique NordEst, dite "OSPAR »). On l'emploie actuellement d'une façon générale pour les traités multilatéraux formels dont les parties sont nombreuses.

Protocole : Acte retenu pour des accords moins formalistes que les conventions et traités. L'instrument peut revêtir différentes formes juridiques.

\section{DROIT COMMUNAUTAIRE (Union européenne)}

Traité : Acte écrit par lequel des États membres établissent des règles créant des droits et obligations et ayant force obligatoire pour les parties au traité (ex. traité EURATOM pour le développement de l'énergie nucléaire en Europe, 1957).

Règlement : Acte juridique de portée générale, obligatoire en tous ses éléments, d'application directe et uniforme dans tous les États membres. Contrairement à la directive, il ne nécessite pas, pour son entrée en vigueur, l'intervention des autorités nationales compétentes.

Directive : Acte juridique adressé aux États, qui fixe des objectifs à atteindre dans un domaine donné et dans un délai prescrit. La directive laisse aux États une « relative » liberté dans le choix de l'acte juridique de transposition en droit national dans la mesure où la directive contient une obligation de résultat et non une obligation de moyen. Si ses termes sont suffisamment clairs et précis, la directive est opposable aux tiers, même à défaut de transposition en droit national dans les délais requis.

Décision : Acte juridique à caractère individuel, applicable à l'État membre, à l'entreprise ou à l'individu visé par elle. Ce sont des mesures individuelles d'exécution qui n'ont pas de portée normative.

Avis et recommandation : Les avis et recommandation expriment une opinion du Parlement européen, ou de la Commission européenne. Ils n’ont pas de portée obligatoire.

Livre vert : Les groupes d'intérêt (ONG...) et les lobbies (entreprises, industriels) prennent une part importante dans le travail préparatoire à l'élaboration de la réglementation européenne. Ce travail peut conduire à la rédaction de livres verts qui rassemblent la réflexion menée dans ce cadre sur un thème donné.

Livre blanc : Le livre vert de réflexion peut déboucher sur un livre banc de stratégie adopté par les instances communautaires. Cette stratégie préfigure le cadre de la nouvelle réglementation européenne du domaine visé (ex. Livre Blanc sur l'Avenir de l’Europe, 2017).

\section{DROIT FRANÇAIS}

Loi : Règle écrite, générale et permanente, élaborée par le Parlement (Assemblée nationale et Sénat), promulguée par le Président de la République et publiée au Journal officiel de la République Française afin qu'elle entre en vigueur.

La loi est applicable à tous, elle définit les droits et les devoirs de chacun. Le domaine qui lui est réservé est délimité par la Constitution (article 34).

Ordonnance : Mesure prise par le pouvoir exécutif (gouvernement), dans un domaine qui relève normalement de la loi. Prévues par l'article 38 de la Constitution, les ordonnances doivent être autorisées par une loi d'habilitation votée par le Parlement, qui en fixe les domaines et la durée. L'ordonnance fait donc partie du domaine 
législatif (une ordonnance peut également désigner une décision rendue par le Président d'un Tribunal ou par un Juge s'il a reçu délégation de celui-ci). L'ordonnance doit être ratifiée par le Parlement.

Décret: Acte réglementaire pris dans un domaine propre ou en application d'une loi pour en définir les modalités d'exécution. Il est signé par le Président de la République ou par le Premier ministre, pour toutes les matières qui ne sont pas expressément réservées à la loi, et est susceptible de recours juridictionnel devant le Conseil d'État (ex. décret d'autorisation création d'une INB).

Arrêté : Acte réglementaire pris par un ou des ministre(s), un préfet ou un maire (ex. arrêté homologuant les décisions de l'Autorité de sûreté nucléaire sur les rejets des centrales nucléaires).

Circulaire : Instructions de service écrites adressées par une autorité supérieure à des agents subordonnés en vertu de son pouvoir hiérarchique. (par ex. circulaire ministérielle, circulaire interministérielle, circulaire préfectorale). Elle contient généralement des instructions, recommandations, ou explications. Elle peut compléter un décret ou un arrêté, mais n'a pas de force obligatoire, étant qualifiée de circulaire interprétative. Néanmoins, dans certains cas, peu nombreux, elle peut avoir un caractère réglementaire lorsqu'elle présente le caractère de circulaire impérative (les circulaires applicables figurent sur le site Internet : www.circulaires.gouv.fr).

Décision individuelle de l'ASN : C'est un acte réglementaire envers l'exploitant d'une INB. Lorsque cette décision fixe les limites de rejet d'effluents, celle-ci est homologuée par le ministre chargé de la sûreté nucléaire. Les décisions sont publiées sur le site Internet de l’ASN (www.asn.fr).

Décision réglementaire de l’ASN : Acte réglementaire pris pour l'application des décrets et arrêtés ; elle est homologuée par le ministre chargé de la sûreté nucléaire.

Jurisprudence : Solution suggérée par un ensemble de décisions suffisamment concordantes rendues par des juridictions judiciaires ou administratives sur une question de droit. La jurisprudence constitue une source du droit.

Code : Ensemble de dispositions normatives faisant partie d'une même branche de droit, placées dans un ouvrage. Toutes les lois ne sont pas codifiées. 


\section{Annexe 5.3}

\section{Code de l'environnement}

Le Code de l'environnement regroupe des textes juridiques relatifs au droit de l'environnement. Les articles qu'il contient sont scindés en deux parties: la partie législative (articles précédés de la lettre L.) ; la partie réglementaire (articles précédés de la lettre R ou D.). Les articles sont structurés en

7 livres et peuvent être facilement consultés sur le site Internet www.legifrance.gouv.fr

\section{Table des matières (susceptible d'évoluer)}

\section{Livre ler : Dispositions communes}

Le premier livre du Code de l'environnement dispose des principes généraux du droit de l'environnement (principes de précaution, pollueur-payeur, etc.) et des objectifs de la nation à ce titre. Il instaure également le principe de l'information et la participation du public aux décisions et projets susceptibles d'impacter l'environnement, par l'institution de procédures d'évaluations environnementales et de consultations locales. Sont mises en place des institutions dont l'objet est de protéger l'environnement et de garantir le respect des principes posés, ainsi que de l'information et la participation du public (OFB, Conseil national de la transition écologique, etc.). Enfin, est visée la police administrative de l'environnement, dont la mission est l'organisation des procédures environnementales et la prévention des dommages causés à l'environnement.

\section{Livre II : Milieux physiques}

Les milieux physiques spécifiquement visés par le Code de l'environnement sont le milieu aquatique, l'air et l'atmosphère.

Le milieu aquatique est surveillé par de nombreuses institutions contrôlant sa qualité et son utilisation. Sont ainsi encadrés par ce Livre II les IOTA (installations, ouvrages, travaux et aménagements sur l'eau) qui disposent d'un régime comparable à celui des ICPE, ainsi que les droits sur les cours d'eau non domaniaux, et de sanctions pénales en cas de non-respect de la réglementation sur l'eau.

La gestion du milieu de l'air porte davantage sur la pollution atmosphérique due aux activités humaines, et sa protection.

\section{Livre III : Espaces naturels}

Les espaces naturels sont protégés et aménagés au regard de leurs spécificités: le littoral est protégé tout en permettant les activités maritimes, les parcs et réserves terrestres ou marins sont des hauts lieux de protection de la nature et la biodiversité mais ont également vocation à accueillir le public, les sites inscrits et classés allient également la protection du patrimoine et l'accueil du public, les paysages sont par nature évolutifs mais sont un volet important de tout projet immobilier. Ce titre évoque également les concepts de trame verte et bleu.

\section{Livre IV : Patrimoine naturel}

La gestion du patrimoine naturel français implique la préservation et la surveillance de ce patrimoine, qu'il soit végétal ou animal, pas le biais notamment de sites de protection comme Natura 2000. Son second volet nécessite la gestion de la ressource animale par l'encadrement de la chasse et de la pêche, qui doit permettre de réguler les populations sauvages, mais garantir en tout point leur sauvegarde voire leur protection intégrale.

\section{Livre V: Prévention des pollutions, des risques et des nuisances}

Le Livre $V$ encadre la majeure partie des activités humaines susceptibles de porter atteinte à l'environnement. Les ICPE (installations classées pour la protection de l'environnement) sont classées selon leur dangerosité et doivent répondre à des prescriptions de l'administration encadrant leur mise en service, fonctionnement, et arrêt définitif. Les produits chimiques dangereux doivent être approuvés pour pouvoir être mis sur le marché ainsi qu'utilisés. Les déchets sont également classés selon leur niveau de dangerosité et doivent être gérés conformément à la réglementation leur étant applicable. Enfin, la sécurité nucléaire et les INB (installations nucléaires de base) font l'objet d'un titre dédié, qui prévoit des institutions de contrôle (ASN), les procédures applicables (création, mise en service, mise à l'arrêt...). Ce titre évoque également les problématiques liées aux ESPN et au transport de substances radioactives.

D'autres aspects des activités humaines sont visés au titre de ce livre, à l'instar de la prévention des nuisances sonores ou des risques naturels (sismiques, volcaniques, etc.).

\section{Livres VI et VII concernent respectivement les territoires d'outre-mer et l'antarctique}

Ce livre vise l'application spécifique des dispositions du Code de l'environnement à la Nouvelle-Calédonie, la Polynésie française, Wallis-et-Futuna, dans les Terres Australes et Antarctiques française, Mayotte et Saint Martin. 


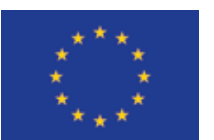

L'Union européenne (UE) a pour institutions le Conseil européen, la Commission, le Parlement, le Conseil de l'UE et la Cour de Justice.

- Le Conseil européen regroupe les chefs d'États ou de Gouvernement des États membres. Il fixe les grandes orientations et donne les impulsions sur les sujets les plus importants mais n'exerce pas de fonction législative. Chaque État membre préside à son tour le Conseil européen pour une période de six mois.

- La Commission européenne est l'institution politiquement indépendante des gouvernements nationaux, qui représente et défend les intérêts de l'UE dans son ensemble. Elle est composée de 27 membres $^{1}$ nommés par les gouvernements. Elle élabore les propositions de nouvelles directives européennes, qu'elle soumet au Parlement européen et au Conseil de l'UE. C'est également le bras exécutif de l'Union. En d'autres termes, elle est responsable de l'exécution des décisions du Parlement et du Conseil.

- Le Parlement européen est composé de représentants des peuples élus au suffrage univer- sel direct tous les 5 ans, et dispose en général d'une compétence législative partagée avec le Conseil de l'UE (procédure de codécision). Dans quelques domaines, par exemple l'agriculture, les politiques économiques, les visas et l'immigration, le Conseil est seul à décider, mais a l'obligation de consulter le Parlement. En outre, l'avis conforme du Parlement est requis pour une série de décisions importantes.

- Le Conseil de l'UE est composé des ministres issus de chaque gouvernement, et représente les États membres de l'UE. Le Conseil de l'UE est le principal organe de décision de l'UE et a pour missions entre autres, d'adopter les législations européennes et de conclure les accords internationaux. Il est aidé dans son travail par le Comité des représentants permanents des États membres (COREPER).

- La Cour de Justice de l'Union européenne (CJUE) est chargée d'assurer le respect du droit dans l'interprétation et l'application des Traités. Elle est composée de juges nommés d'un commun accord par les États membres.

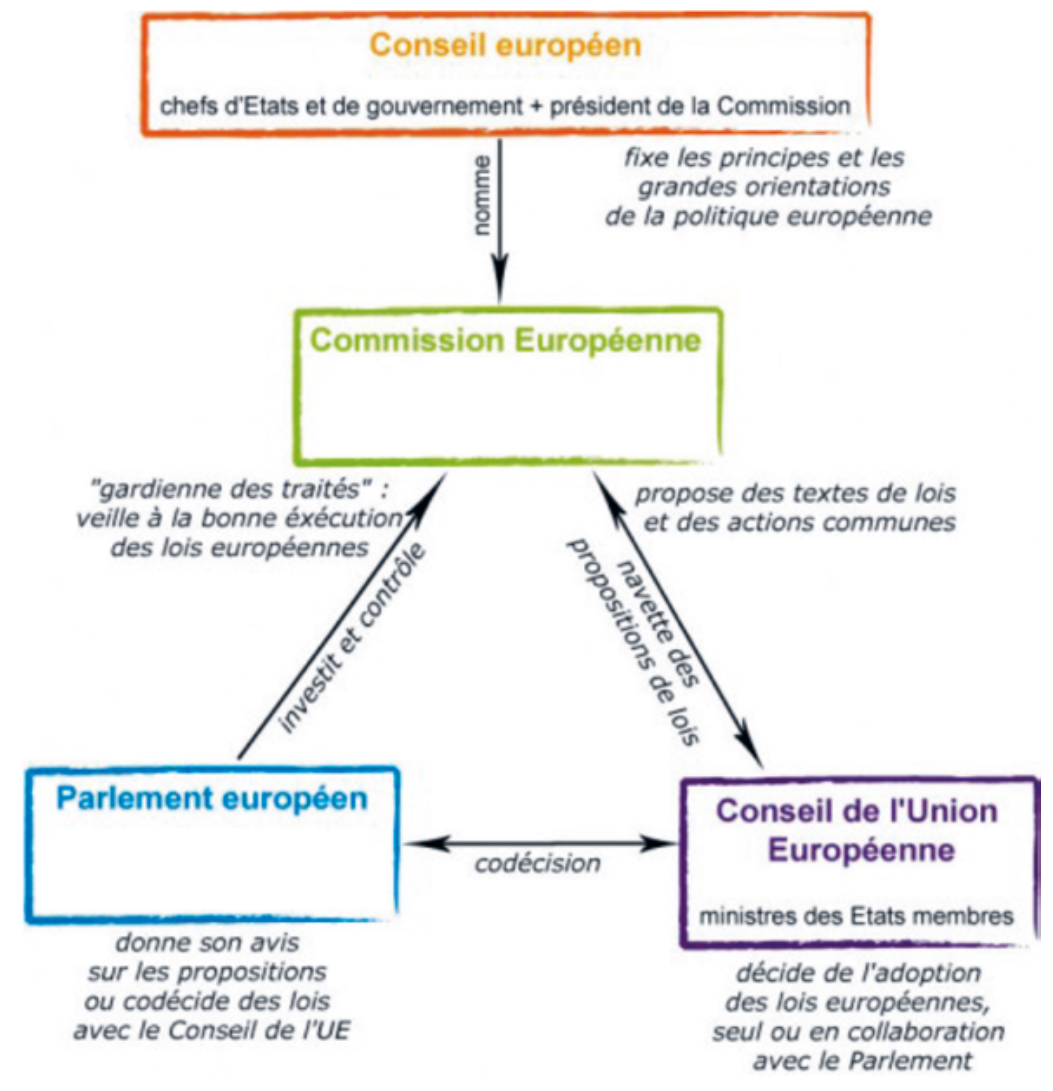

1. Suite au retrait du Royaume-Uni de I'UE. 
4.

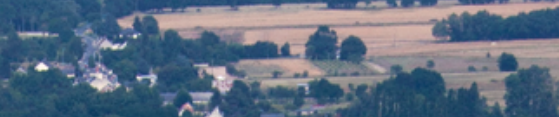

$$
\begin{aligned}
& \text { is: }
\end{aligned}
$$

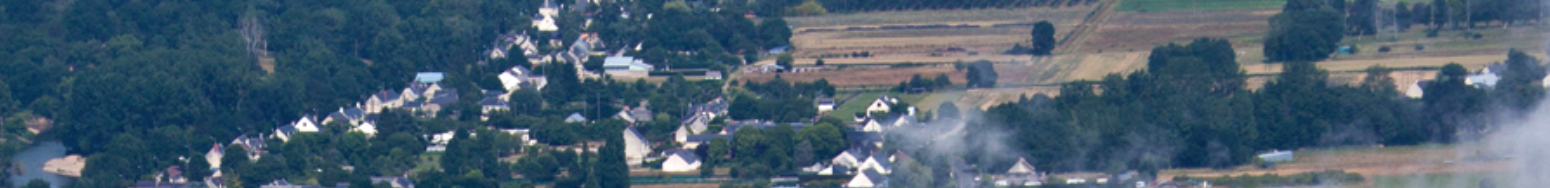

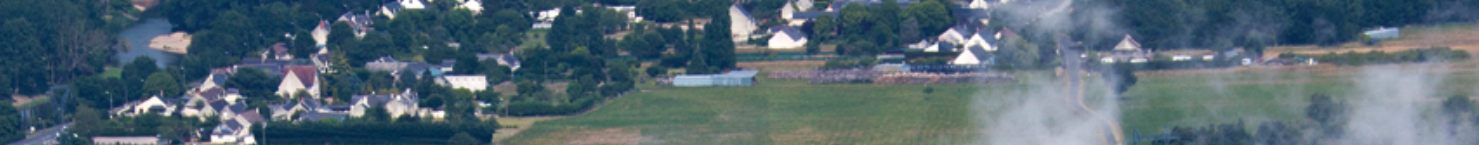

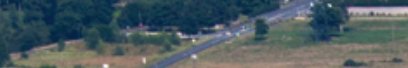

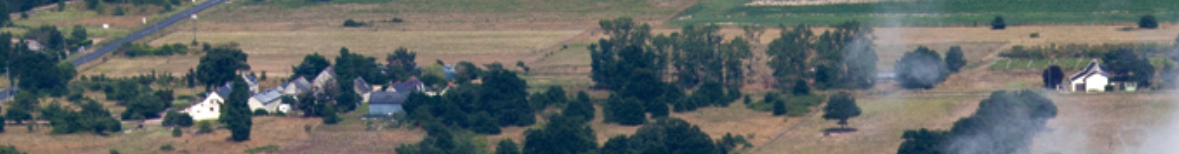

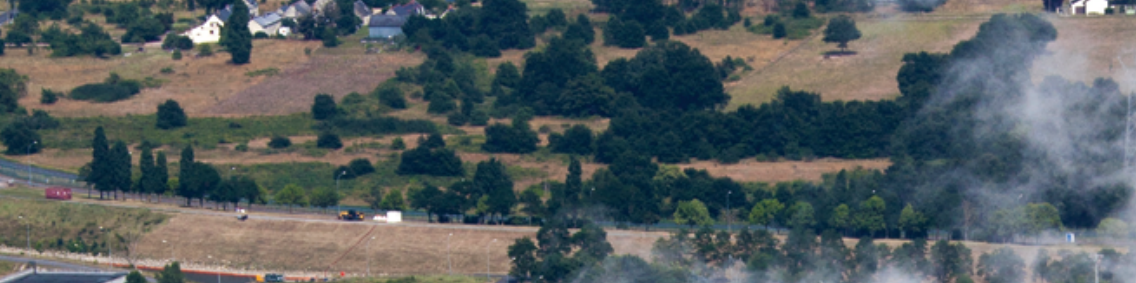

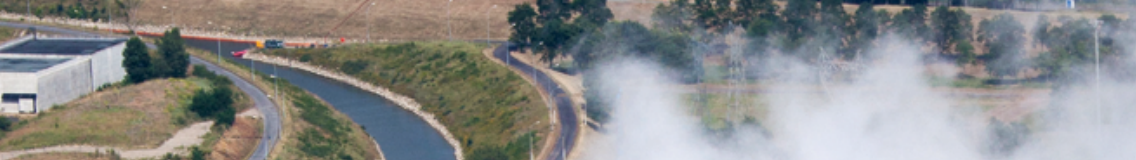

(2)

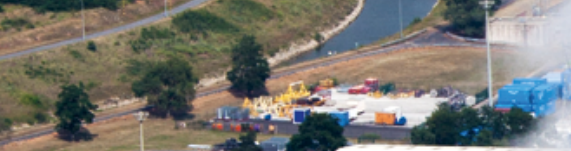

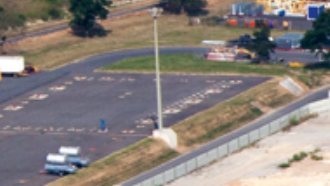

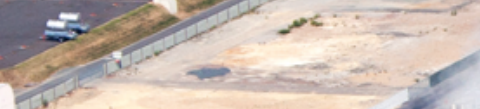

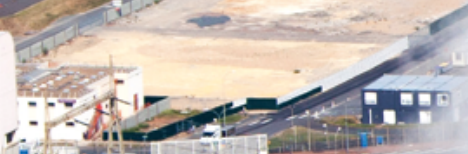

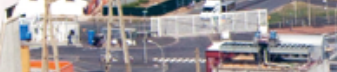

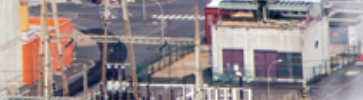

2(25)

$[2)^{2}:-$

+

So 